\title{
Zur Kenntnis der Entwicklungsanomalien bei Infantilismus und bei vorzeitiger Geschlechtsreife ${ }^{1}$.
}

\author{
Von \\ Dr. Bruno Wolff in Berlin.
}

(Mit 4 Textfguren.)

In den letzten Jahren haben die Aerzte sich vielfach mit dem Studium des Infantilismus und Foetalismus beschäftigt. Wenn die Aufmerksamkeit auf dieses Gebiet mehr als zuvor gelenkt wurde, so war dies in erster Linie A. Hegar $(27,28,29,30,31)$ und W. A. Freund $(19,20,21)$ zu verdanken. Hauptsächlich an die grundlegenden Arbeiten dieser beiden Forscher haben sich die neueren Untersuchungen über Infantilismus angeschlossen.

Das klinische Interesse, das die Lehre vom Infantilismus erweckt, findet seine Berechtigung vor allem in der besonders von W. A. Freund klar erkannten Tatsache, dass in dem Infantilismus „eines der bedeutsameren, prädisponierenden Momente für die Ausbildung pathologischer Züstände" $\mathrm{zu}$ erblicken ist [Chrobak und v. Rosthorn (13)]. "Vieles, das man sonst mit dem Wort ,Disposition" abzufertigen pflegt", gewinnt, wie Chrobak und v. Rosthorn (13) in ihrem ausgezeichneten Buche über „die Missbildungen der weiblichen Geschlechtsorgane" mit Recht hervorheben, durch die Lehre vom Infantilismus "eine greifbare Basis".

Nicht minder gross als das klinische Interesse ist das allgemein biologische.

So ist die Frage nach der Entstehung der sekundären Geschlechtscharaktere mit der Lehre vom Infantilismus oder oder wenigstens mit demjenigen Teile dieser Lehre eng verknüpft, der sich auf die infantilen Entwickelungsstörungen des Genitalapparates bezieht.

1) Zum Teil vorgetragen in der Sitzung der Gesellschaft für Geburtshilfe und Gynäkologie zu Berlin am 10. März 1911. 
Im weiteren sind die Erfahrungen in Fällen von Infantilismus ganz allgemein von Wert für das biologisch so wichtige Gebiet der Lehre von den Korrelationen der einzelnen Organe untereinander ${ }^{1}$ ), speziell für das Studium der Wechselwirkungen der Organe mit innerer Sekretion.

Bei der Bedeutung, die der Lehre vom Infantilismus zukommt, schienen mir vier Be obachtungen, im Zusammenhang miteinander, der Mitteilung wert zu sein, in denen bei weiblichen Individuen im Alter zwischen 19 und 45 Jahren die Pubertätsentwickelung vollständig oder nahezu vollständig ausgeblieben war und zugleich noch andere symptome von Infantilismus bestanden.

Als ein Gegenstück zu diesen Beobachtungen teile ich in der vorliegenden Arbeit ausserdem einen Fall mit, in dem bei einem erst vierjährigen Kinde Menstruation und sonstige Zeichen der Geschlechtsreife vorzeitig sich eingestellt hatten.

Die Fälle 1, 4 und 5 entstammen dem Material der gynäkologischen Poliklinik des Krankenhauses der jüdischen Gemeinde zu Berlin. Die Fälle 2 und 3 verdanke ich Herrn Professor H. Strauss, der sie mir aus der inneren Abteilung des Krankenhauses gütigst überwiesen hat.

Die Röntgenuntersuchungen, über die ich berichten werde, hat, auf meine Veranlassung, Herr Professor Dr. Levy-Dorn ausgeführt, und die dabei gewonnenen Bilder sind von ihm begutarhtet worden. Ich spreche ihm dafür an dieser Stelle meinen besten Dank aus.

In den Fällen 1 und 4 habe ich, gemeinsam mit Herrn Dr. Leo Zuntz, auch Untersuchungen über den respiratorischen Stoffwechsel and die Einwirkung von Oophorin auf diesen Stoffwechsel vorgenommen. Auf diese Versuche gehe ich im folgenden nicht weiter ein, da L. Zuntz darüber an anderer Stelle des genaueren berichten wird.

Meine Beobachtungen sind die folgenden:

1) Vergl. hierzu die neueren umfassenden Darlegungen von v. Hansemann (26) über den "Altruismus" der Zellen. v. Hansemann bat dabei mehrfach auf den Infantilismus besonders Bezug genommen. 


\section{Vollständiges oder fast vollständiges Ausbleiben der Pubertätsentwickelung.}

Fall 1 (siehe Fig. 1). Frl. 0. kommt am 20. VII. 1908 zum ersten Mal in die Poliklinik. Die Patientin ist im August 1888 geboren, also fast $20 \mathrm{Jahre}$ alt.

Figur 1.

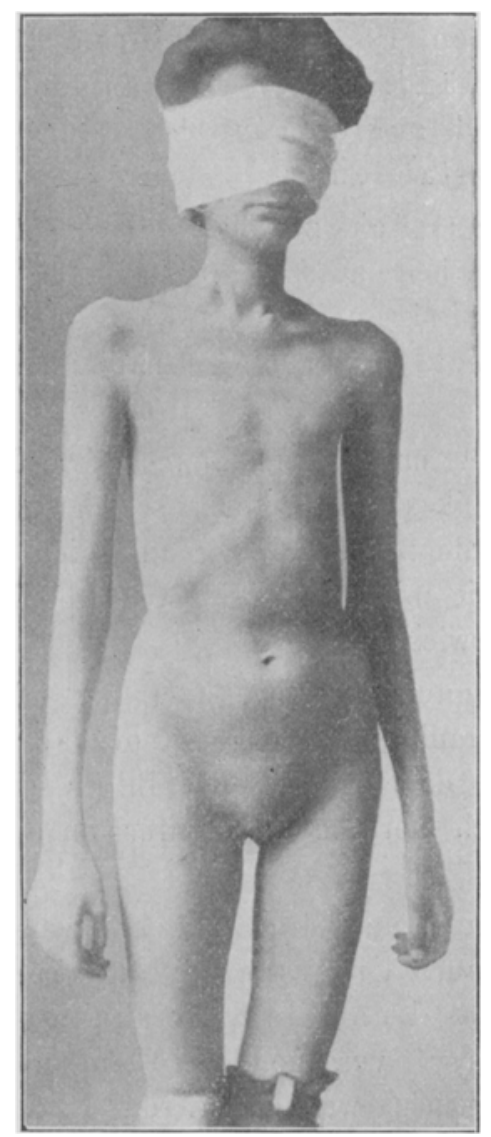

Sie ist angeblich ${ }^{1}$ ) rechtzeitig und normal entwickelt zur Welt gekommen. Schon als Kind soll sie immer schwächlich und zart gewesen sein, im übrigen ist während ihrer Kinderzeit in ihrer körperlichen und geistigen Entwickelung nichts Besonderes aufgefallen.

1) Die Angaben der Patientin selbst wurden ergänzt durch Angaben ihrer Mutter. 
Nennenswerte Krankheiten, abgesehen von Keuchhusten, soll sie nicht durchgemacht, insbesondere keine Rachitis und keine Lungenkrankheiten gehabt haben. Ihre Mutter (vgl. unten den Untersuchungsbefund bei der Mutter) hat ausser dieser Tochter noch 2 Töchter und einen Sohn geboren. Die Mutter hat keines ihrer Kinder genährt. Die anderen Kinder sollen normal entwickelt sein (vgl. unten).

Die Patientin hat niemals die Menses oder an deren Stelle auftretende Beschwerden gehabt. Sie hat zur Zeit überhaupt keine körperlichen Beschwerden, sondern kommt in die Poliklinik nur, um die Ursache der Amenorrhoe zu erfahren.

Es handelt sich um ein äusserst dürftig entwickeltes, sehr mageres Mädchen von $151 \mathrm{~cm}$ Körperlänge, einem Gewicht von $31,0-31,5 \mathrm{~kg}$, äusserst zartem Knochenbau.

Die oberen Extremitäten sind von aufallender Länge. An den Beinen bemerkt man eine Andeutung von Genu valgum.

In geistiger Beziehung macht die Patientin einen mädchenhaften, schüchternen, aber durchaus in telligenten, ihrem Alter und Bildungskreis entsprechend entwickelten Eindruck.

Der Gehirnschädel zeigt eine auffallend quadratische Form und ist ziemlich gross im Verhältnis zum Gesichtsschädel; der Umfang des Kopfes beträgt $54 \mathrm{~cm}$. Die Gesichtsbildung zeigt nichts Besonderes. Der Gaumen ist hoch und spitzbogenförmig, die Zähne sind defekt, einige Zähne schiefgestellt. Das Haupthaar ist voll entwickelt, von gewöhnlichem weiblichen Typus; es soll früher sehr lang gewesen, später zum Teil ausgegangen sein. Das Gesicht zeigt keine abnorme Behaarung, insbesondere keine virilen Merkmale (keine Bartbildung).

Die Schilddrüse ist vorhanden, aber klein.

Der Thorax zeigt etwas langgestreckte, schmale Form. Die Supraund Infraclaviculargruben sind mässig vertieft. Schulterumfang $80 \mathrm{~cm}$.

Thoraxumfang über den Mamillae gemessen: bei Inspiration $63 \mathrm{~cm}$, bei tiefster Exspiration $61 \mathrm{~cm}$. Unter den Achselhöhlen fehlt, abgesehen von 2-3 dünnen Härchen, jegliche Behaarung. Brust und Rïcken sind ohne Behaarung. An normaler Stelle bemerkt man ganz rudimentär entwickelte Mammillen, die an Grösse kaum den gewöhnlichen Brustwarzen eines Mannes entsprechen. Die Mammillae haben einen Durchmesser von etwa $2 \mathrm{~mm}$ und ragen kaum $1 \mathrm{~mm}$ über die Oberflächie hervor. Sie sind von ganz blassen Warzenhöfen umgeben, von denen der linke $1,5 \mathrm{~cm}$, der reehte $1,7 \mathrm{~cm}$ im Durchmesser misst. Im übrigen ist keinerlei Andeutung einer Mamma zu konstatieren.

Die Atmung ist sehr wenig ausgiebig, über der rechten Lunge vorn etwas rauhes Exspirium; im übrigen ist der Lungenbefund ohne Besonderheit.

Cor: Reine Herztöne; Herzdämpfung zeigt keine deutliche $\mathrm{Ab}$ weichung vom Gewöhnlichen: Spitzenstoss bis etwa 1 Querfinger weit nach links von der Mammillarlinie verbreitert (wahrscheinlich Dilatation des linken Ventrikels in Folge Enge der Aorta).

Bauch, Unterleibsorgane: Entfernung vom Nabel zur Symphyse: $16 \mathrm{~cm}$. Um die Genitalien einige minimale Härchen, im übrigen fehlt am Bauch und in der Schamgegend jede Spur einer Behaarung.

Genitalien: Die äusseren Genitalien sind weiblich und sonst vollkommen normal entwickelt, sie entsprechen aber in ihrer Ausbildung nur den Geschlechtsteilen eines erst. etwa 8 bis 
9 jährigen Kindes. Die grossen Labien sind ganz flach, die kleinen Labien sind vorhanden, aber sehr klein, die Clitoris ist gleichfalls sehr klein. Das Hymen ist als dünne Membran deutlich ausgebildet, zeigt keinen Einriss und hat eine etwa $3 \mathrm{~mm}$ breile Oeffnung. Die Schleimhänte sind succulent (wie beim Kinde, nicht atrophisch, wie im senilen Zustand). Die Entfernung von der Clitoris bis zum Frenulum labiorum beträgt etwa $21 / 2 \mathrm{~cm}$. Der Damm ist schmal, nur etwa $2 \mathrm{~cm}$ lang. Durch die Hymenalöffnung kann man in die Vagina einen Glaskatheter $7-71 / 2 \mathrm{~cm}$ weit ohne Schwierigkeit einführen; dann kommt man auf Widerstand. Digitale vaginale Untersuchung ist bei der Enge der Teile nicht ausführbar. Untersucht man per rectum und führt gleichzeitig einen Katheter in die Vagina, so weit wie möglich, ein, so fühlt man, von der Spitze des Katheters ausgehend, einen feinen, nach oben hin etwas dicker werdenden Strang, der etwa $5 \mathrm{~cm}$ lang und an seinem vaginalen Ende etwa $1 \mathrm{~mm}$, weiter oben etwa $2-3 \mathrm{~mm}$ dick ist. Dieser Strang könnte wohl dem Uterus entsprechen (mit Sicherheit lässt sich dies allerdings nicht entscheiden). Den Ovarien entsprechende Körper lassen sich nicht auffinden.

Harnapparat: Urin klar, enthält weder Eiweiss noch Zucker. Urethralöffnung an normaler Stelle, ziemlich eng. Mit einem Kindercystoskop wird, nach subkutaner Einspritzung ron Indigcarmin, die Cystoskopie vorgenommen; sie ergibt: auffallend zarte Blasenschleimhaut, am Blasenboden sehr reichliche feine Gefässe, keine Rötung, keine Trübung. Man bemerkt, nahe beieinander liegend, deutlich rechts 2 kreisrunde Ureteröffnungen und links eine spaltförmige, feinere Oeffnung. Die beiden rechtsseitigen Ureteröffnungen und die linksseitige stehen auffallend nahe beieinander. Die Blasenkapazität ist gering; bei Füllung der Blase mit $250 \mathrm{ccm}$ Flüssigkeit empfindet die Patientin schon starken Harndrang.

Becken: Umfang des Beckens über den Spinae ant. sup. gemessen: $65^{1} / 2 \mathrm{~cm}$, über dem Mons veneris gemessen $70^{1 / 2} \mathrm{~cm}$. Diam. spin.: $221 / 2 \mathrm{~cm}$, D. crist.: $24 \mathrm{~cm}$, D. troch.: $27 \mathrm{~cm}$, Conj. ext.: $161 / 2 \mathrm{~cm}$. (Das Promontorium zu erreichen, gelingt bei der mit einem Finger per rectum vorgenommenen Untersuchung nicht.)

Nates flach, wenig gerundet.

Röntgenuntersuchung: Die Röntgenuntersuchung der rechten Hand (siehe Fig. 2) ergibt an allen Phalangen und Metacarpalknochen sowie an der unteren Radius- und Ulnaepiphyse offene Epiphysenlinien. Der Knochenbau entspricht dem 15. Lebensjahr ${ }^{1}$ ). Durchleuchtung des Beckens ergibt an der oberen Femurepiphyse of fene Epiphysenlinien.

Weiterer Verlauf: Pat. wird mit Oophorin (Näheres siehe in der Arbeit von L. Zuntz) und allgemein roborierenden Mitteln behandelt und bis zum 1. Juni 1909, also fast ein Jahr lang, in der Poliklinik beobachtet. Dann verlässt sie Berlin. Sie sieht bei der

1) Vgl. hierzu die Abbildungen in dem Atlas von Wilms und Sick (60). Wilms sagt: „Im 17. oder Anfang des 18. Jahres verschwinden in der Regel die Epiphysenlinien der Finger und Metacarpalknocben und erst Ende des 18. Jahres"...., verstreichen auch die Epiphysenlinien am unteren Ende von Ulna und Radius". 
Entlassung im ganzen frischer und etwas kräftiger aus; im übrigen ist eine Veränderung des Zustandes nicht zu konstatieren.

Auch die Mutter und eine Schwester der Patientin konnten gelegentlich in der Poliklinik untersucht werden: Die Mutter ist 42 Jahre alt, hat regelmässig die Menses, hat, wie schon oben erwähnt, 4 Kinder geboren, keines selbst genährt. Die Untersuchung ergab: Prolapsus recti, Metritis, Retroflexio uteri mobilis (grosser derber Uterus). Mammae schwach entwickelt. Befund sonst ohne Besonderheit.

Figur 2.

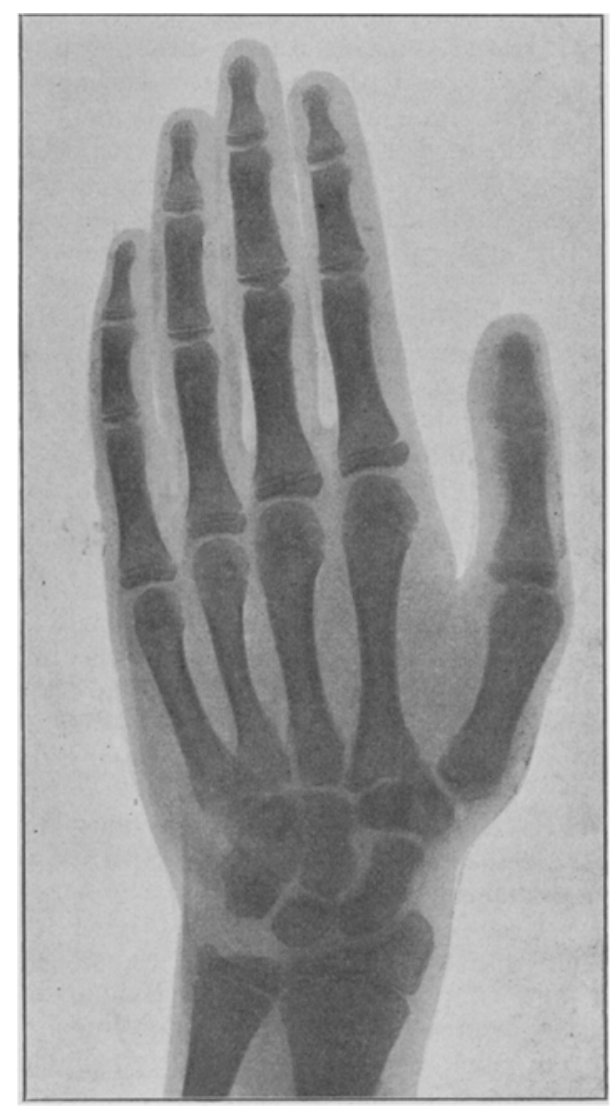

Bei der 13jahrigen, noch nicht menstruierten Schwester der Patientin ergab die Untersuchung mit Wahrscheinlichkeit Tuberculosis pulmonum.

Beobachtung 2.1) Fräulein P., kommt am 4. Juli 1910 zum ersten Male in die Poliklinik. Sie ist 19 Jahre alt. Der Vater soll

1) Unter Mitbenutzung des Krankenjournals der inneren Abteilung des Krankenhauses. 
an Lungenkrankheit gestorben, eine Schwester herzkrank sein. Die Patientin hat als Kind Masern und Typhus gehabt. Seit einigen Wochen leidet sie an Kopfschmerzen, seit ca. 8 Tagen an Magenschmerzen. Deshalb wurde sie in die innere Abteilung des jüdischen Krankenhauses aufgenommen. Die Untersuchung ergab dort eine "Gastritis anacida“. Patientin hat mit Erfolg die Bürgerschule durchgemacht und danm kochen gelernt.

Die Menses sind noch nie eingetreten, periodische Schmerzen (an Stelle der Menses) treten nicht auf.

3 Schwestern der Patientin haben die Menses zur gewöhnlichen Zeit bekommen.

Die Patientin macht den körperlichen Gesamteindruck eines wenig kräftigen, etwa $13 \mathrm{Jahre}$ alten Mädchens. Sie ist $150 \mathrm{~cm}$ lang, von zartem Knochenbau. Geringer Grad von Dorsalskoliose, sonst keine Anzeichen für überstandene Rachitis. Eine ungewöhnliche Länge der oberen Extremitäten ist augenfälilig.

In geistiger Beziehung macht die Patientin einen ihrem Alter entspreehenden, intelligenten, psychisch normal entwickelten, mädchenhaften Eindruck.

Sie hat gut entwickeltes Haupthaar von weiblichem Typus. Die Gesichtsziuge sind ausgesprochen kindlich (mädchenhaft). Die Schilddrüse ist auffallend klein, nur undeutlich zu fühlen. Der Gaumen ist deutlich spitzbogenförmig.

Der Thorax zeigt kindliche Form. Axillarhaare fehlen vollständig. Auf Brust und Rücken keine Behaarung. Die Mammillae, an gewöhnlicher Stelle, sind kaum linsengross, haben blasse, 1 bis $2 \mathrm{~cm}$ breite Warzenhöfe; im übrigen fehlt jede nachweisbare Spur von Brustdrüsen. Die Lungen ergeben überall normalen Perkussionsschall, überall reines vesikuläres Atmen. Cor: Normale Grenzen. Spitzenstoss an normaler Stelle. Reine, regelmässige Herztöne. Puls beiderseits gleichmässig, regelmässig. voll.

Abdomen flach, weich, ohne Besonderheit. Magenuntersuchung (Befund der inneren Abteilung) ergab Fehlen freier Salzsäure. Nieren nicht palpabel.

Am Mons veneris einige wenige, $3-4 \mathrm{~cm}$ lange Haare. Im übrigen fehlt in der Schamgegend jegliche Spur einer Behaarung. Bauch zeigt keine Behaarung.

Genitalien: Grosse Labien ganz flach, kleine Labien sehr wenig entwickelt. Hymen intact, zeigt infolge eines sagittal verlaulenden dünnen Septums eine doppelte Oeffnung. (Hymen biperforatus.) In die verhältnismässig weite Vagina kann man einen Glaskatheter ca. $7 \mathrm{~cm}$ weit einführen. Bei der per rectum vorgenommenen digitalen Untersuchung fühlt man sehr undeutlich einen ca. $3 \mathrm{~cm}$ langen dünnen Strang, der dem Uterus entsprechen könnte; mit Sicherheit lässt sich dies allerdings nicht entscheiden. Ovarien zu fühlen gelingt nicht. Der Damm ist klein, muldenförmig, ca. $2-3 \mathrm{~cm}$ lang.

Beckenmasse: Diam. spin.: 21, Diam. crist.: 24, Diam. troch.: 25, Conj. ext.: $17 \mathrm{~cm}$.

Röntgenuntersuchung: Die Röntgenuntersuchung der rechten Hand ergibt an den Phalangen und Metacarpalknochen sowie an der unteren Radius- und Ulnaepiphyse offene Epiphysenlinien. Der 
Knochenbau entspricht etwa dem Aiter von 15-16 Jahren. (Vgl. oben Seite 546, Anm. 1.)

Das Röntgenbild der Schädelbasis lässt eine Erweiterung der Sella turcica nicht erkennen, (also keine nachweisbare Vergrösserung der Hypophysis). Die Sella turcica ist eher eng als weit.

Beobachtung $3^{1}$ ). Fräulein M. kommt zum ersten Male am 15. VIII. 1910 in die Poliklinik. Sie ist 45 Jahre alt.

Patientin gibt folgendes an: Ihre Mutter ist an "Herzlähmung" gestorben. Ihr Vater lebt noch. Lungenkrankheiten sind in der Familie nicht vorgekommen. Der Vater ist von normalem Wuchs; die Mutter war sehr klein (aber keine Zwergin). Alle anderen Familienmitglieder sollen von normaler Grösse sein. Sie selbst sei von ihrer Mutter an der Brust genährt worden. Schon als Kind sei sie klein und schwächlich gewesen; im Alter von 4 Jahren musste sie noch getragen werden. Etwa von ihrem 8. Lebensjahre an sei ihre Kleinheit besonders aufgefallen. Sie glaubt, als Kind skrofulös gewesen zu sein und englische Krankheit und Masern gehabt zu haben. In ihrem 18. Jahre hatte sie eine schwere Influenza; im Anschluss daran bemerkte sie, dass ihr das Gehen und Steigen schwer wurde. Vor 18 Jahren hatte sie rheumatisches "gastrisches" Fieber. Häufig litt sie an Bronchialkatarrh. Seit vielen Jahren fühlt sie sich schwach und bemerkt, dass Lippen, Nägel und Gesicht, besonders nach Anstrengungen, blau aussehen. Sie klagt ausserdem über Herzklopfen, Kopfschmerzen und Schwindelgefühl.

Von Interesse ist aus der Anamnese weiterhin folgendes: Patientin gibt an, die Elementarschule mit Erfolg durchgemacht zu haben, im Rechnen sei sie schwach, in den übrigen Fächern aber tadellos gewesen. Später war sie als Sängerin und Schauspielerin bei einer sogenannten Liliputanertruppe angestellt. In dieser Zeit, (1882 bis 1895) also im Alter von 17 bis 30 Jahren sei sie noch sehr gewachsen. (Die gleiche Beobachtung sei auch bei den anderen Mitgliedern jener Liliputanertruppe gemacht worden. [Vgl. unten Seite 572, Joachimsthal (37)]. Ob sie jetzt noch wächst, weiss sie nicht.

Sie hat niemals die Menses oder vikarierende Erscheinungen bekommen.

Die Patientin ist von zwerghaft kleiner Gestalt (Körperlänge $120 \mathrm{~cm}$ ), geringem Fettpolster, zartem Knochenbau. Der Körper zeigt eckige Formen und macht einen knabenhaften Eindruck. Es besteht eine mässige rechtsseitige Dorsokyphoskoliose und mässige Lordose der Lendenwirbelsäule.

Sowohl die Arme wie die Beine erscheinen verhältnis$\mathrm{mässig} \mathrm{lang.} \mathrm{(Länge} \mathrm{der} \mathrm{Arme} 55 \mathrm{~cm}$, Länge der Beine vom Trochanter an $66 \mathrm{~cm}$.)

Die Haut ist von normaler Farbe. Die sichtbaren Schleimhänte sind dunkelbläulich verfärbt.

In psychischer Hinsicht macht die Patientin einen sehr intelligenten, durchaus ihrem Alter entsprechenden, mädchenhaften Ein-

1) Unter Mitbenutzung des Krankenjournals der inneren Abteilung des Krankenhauses. 
druck. Was ihr sexuelles Empfinden anbetrifft, so gibt sie an, sie sei in dieser Hinsicht ganz "kindlich".

Das Haupthaar ist glatt und kurzgeschnitten (wie Patientin sagt deshalb, weil sie als Schauspielerin eine Perrücke trägt; wenn sie das Haar nicht abschnitte, würde es, wie sie angibt, lang gewachsen sein). Die Gesichtszüge sind kindlich. Die Stimme ist sehr hoch und, wie Patientin selbst angibt, sehr kräftig. Sie singe mit Leichtigkeit das hohe $\mathrm{C}$ und sogar noch einen Ton darüber. Der Gaumen ist flach. Schilddrüse nicht mit Sicherheit zu fühlen, jedenfalls sehr klein.

Figur 3.

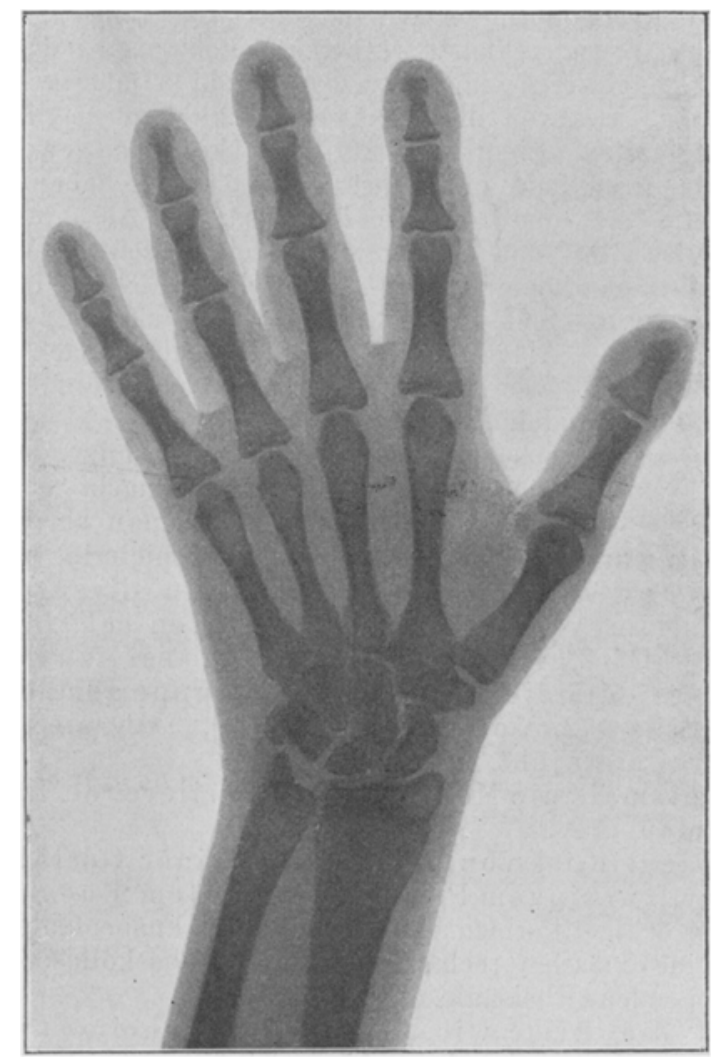

Thorax von etwas gedrungener Gestalt. Keine Spur von Axillarhaaren. Mammaekaum angedeutet; Mammillae fehlen fast vollständig. (Links ist die Mammilla kaum angedeutet, die rechte Mammilla entspricht an Grösse etwa dem vierten Teile einer Linse.) Ganz blasse, $1-1 \frac{1}{2} \mathrm{~cm}$ breite Warzenhöfe.

Lungen: (Befund der inneren Station): Ueberall normaler Perkussionsschall. Untere Grenzen mässig verschieblich. Ueber der rechten Lungenspitze zuweilon einige Rhonchi auf der Höhe der Inspiration, 
sonst überall reines vesikuläres Atmen. Herz: (Befund der inneren Station): Die absolute Dämpfung reicht rechts bis zur Mitte des Sternums und oben bis zur dritten Rippe, nach links einen Querfinger breit über die Mamillarlinie; Spitzenstoss im unteren Interkostalraum etwas ausserhalb der Mamillarlinie. Töne an der Spitze leise, regelmässig. Erster Ton leicht gespannt. Ueber der Aorta ist der zweite Ton accentuiert. Ueber der Pulmonalis ist ein systolisches Geräusch hörbar. Aenssere Untersuchung des Abdomens ergibt nichts Besonderes. In der Schamgegend keine Spur einer Behaarung. Die äusseren Genitalien machen den Gesamteindruck der normal gebildeten Geschlechtsteile eines $2-3$ jährigen Mädchens. Die Länge der Vulva beträgt ca. $2 \mathrm{~cm}$. Die grossen Labien sind sehr flach, die kleinen Labien und die Clitoris sind angedeutet. Der Hymen zeigt einen nur $1-2 \mathrm{~mm}$ breiten Saum, mit einer Oeffnung, in die man einen Glaskatheter bequem $6 \mathrm{~cm}$ weit hineinführen kann.

Die Urethralöffnung befindet sich an der gewöhnlichen Stelle.

Der Damm ist sehr eng, ca. $3 \mathrm{~cm}$ lang.

Anus und Rectum sind sehr eng, wie bei einem Kinde; dadurch ist die rectale Untersuchung erschwert. Bei der rectalen Untersuchung, die bei der Enge der Teile nur sehr schonend vorgenommen werden kann, ist von den inneren Genitalien nichts festzustellen.

Im Urin zeitweilig Albumen.

Beckenmaasse: Diam. spin.: $191 / 2 \mathrm{~cm}$, Diam. crist.: 22, Diam. troch.: 23, Conjug. ext.: $151 / 2 \mathrm{~cm}$.

Röntgenuntersuchung: Die Röntgenuntersuchung der rechten Hand (siehe Fig. 3) einschliesslich des Handgelenks ergibt, dass die Epiphysenlinien an der unteren Ulna- und Radiusepiphyse noch offen, an den anderen Knochen aber verschwunden sind. Das Bild entspricht dem 18. Lebensjahr. (Vgl. oben Seite 546, Anmerkung 1.)

Die Röntgenuntersuchung der Schädelbasis lässt eine Verbreiterung der Sella turcica nicht erkennen, (keine Vergrösserung der Hypophysis nachweisbar).

Beobachtung 4. Fräulein B. kommt am 18. VIII. 1908 zum ersten Male in die Poliklinik, ist $193 / 4$ Jahre alt. Patientin gibt an, als Kind sehr schwächlich gewesen zu sein, sodass sie mehrmals auf längere Zeit die Schule versäumen musste. Englische Krankheit will sie nicht gehabt haben.

Sie klagt über allgemeine Mattigkeit. Die Menses sind noch niemals eingetreten.

Patientin ist von kleiner Statur, $151 \mathrm{~cm}$ lang. Gewicht (am 15. X. 08) $46 \mathrm{~kg}$. Fettpolster ziemlich gut entwickelt, geringer Grad von Adipositas.

Es besteht eine mässige rechtsseitige Kyphoskoliose der Brustwirbelsäule. Leichter Grad von Pectus carinatum.

Sehr blasse Schleimhänte.

In psychischer Hinsicht macht die Patientin einen mädchenhaften Eindruck, Intelligenz von der gewöhnlichen, dem Alter entsprechenden Entwicklung. Ueber ihr sexuelles Empfinden befragt, gibt Pat. an, dass sie "kalt" sei. 
Das Haupthaar ist voll entwickelt, zeigt den gewöhnlichen weiblichen Typus.

Brustumfang direkt unterhalb der Mammae $77 \mathrm{~cm}$, gerade über den Mamillae gemessen $80 \mathrm{~cm}$. Die Mammae sind äusserst gering entwickelt, das Volumen des Drüsenkörpers der Brüste ist $z u$ sehätzen auf nicht mehr als Wallnussgrösse, der Drüsenkörper stellt sich dar als eine flache Scheibe. Der Bogen, den die Mammae, über den Mammillen gemessen, bilden, misst $8 \mathrm{~cm}$. Die Mammillen sind beiderseits kaum mehr als linsengross. Der Warzenhof wird durch einen blassen, etwa $1 \mathrm{~cm}$ breiten Streifen gebildet.

Unter den Achselhöhlen ist die Beharung beiderseits minimal, der Haarstreif ist hier an jeder Seite nur $1 \frac{1}{2} \mathrm{~cm}$ breit, dabei links $3 \mathrm{~cm}$, rechts $5^{1} / 2 \mathrm{~cm}$ lang.

Lungenbefund ohne Besonderheit. Cor: Reine, aber leise Herztöne, Spitzenstoss nicht verbreitert, Herzdämpfung wie gewöhnlich.

In der Schamgegend nur sehr mangelhafte Behaarung von weiblichem Typus. Die äusseren Genitalien machen einen infantilen Eindruck. Die kleinen Labien sind etwas elongiert. Clitoris vorhanden. Hymen intakt. Hymenalöffnung sehr eng. Aus der Vagina kommt geringer weisslicher Fluor. Bei der rektalen Untersuchung fühlt man deutlich einen sehr kleinen, etwa $3 \mathrm{~cm}$ langen, $1-1 \frac{1}{2} \mathrm{~cm}$ breiten, dem Uterus entsprechenden Körper. Dagegen gelingt es nicht, die Ovarien zu fühlen. Urin, mittels Katheters entleert, klar, frei von Eiweiss und Zucker. Harnröhrenmündung an normaler Stelle.

Beckenmaasse: Diam. spin.: $25 \frac{1}{2} \mathrm{~cm}$, Diam. crist.: $26 \mathrm{~cm}$, Diam. troch. : $29 \mathrm{~cm}$, Conj. ext.: $18^{1} / 2-19 \mathrm{~cm}$.

Röntgenuntersuchung: Die Röntgenuntersuchung der rechten Hand ergibt an den Phalangen und Metacarpalknochen sowie an der unteren Radius- nnd Ulnaepiphyse offene Epiphysenlinien, Verhältnisse, wie sie dem 16. Lebensjahre entsprechen (vgl. S. 546 Anm.).

Pat. wird mit 0ophorin behandelt. Eine objektiv nachweisbare Veränderung des Zustandes tritt während der Beobachtungszeit nicht ein.

\section{Vorzeitige Entwickelung der Geschlechtsreife.}

Beobachtung 5. 4 Jahre und 1 Monat altes Mädchen kommt mit der Mutter am 5. IX. 10 zum ersten Male in die Poliklinik.

Die Nutter des Kindes ist von ziemlich kleiner Statur, zart gebaut, hat die Menses mit 15 Jahren bekommen, hat nur dieses eine Kind geboren.

Die Eltern des Kindes sind blutsverwandt, und $z$ war sind dieselben Kinder von Stiefschwestern.

Das Kind soll schon bei der Geburt stark entwickelt gewesen sein, die Hebamme soll bei der Geburt gesagt haben, es sei „wie ein Vierteljahrkind". Es war dann immer kräftig. Bis zum 4. Monat wurde es ausschliesslich an der Brust der Mutter ernährt, dann bekam es die Brust und die Flasche. Die ersten Zähne sollen im Alter von 7 Monaten, Backzähne im Alter von 1 Jahr durchgebrochen sein.

Seit 2 Jahren, also etwa yon Beginn des 3 . Lebensjahres des Kindes an, bemerkte die Mutter das periodische Auftreten von Blutungen aus den Geschlechtsteilen des Kindes. In der letzten Zeitist genau notiert worden, wann solche Blutungen 
sich einstellten, und zwar war dies der Fall am 3.-6. Februar, 2.-9. März, 21.-25. April, 18.—20. Mai, 28.-30.Juli, 27.-29. August.

Wenn man das Kind in Kleidern sieht, glaubt man auf den ersten Blick, dass es sich um ein etwa $7 \mathrm{jähriges}$ Mädehen handelt. Das Kind hat regelmässige Gesichtszüge, auffallend langes (38 cm), fast bis an die Hüften herabreichendes Haupthaar. Es ist $\left.121 \mathrm{~cm} \mathrm{lang}^{1}\right), 26 \mathrm{Kil}$ schwer. Die Spannweite der Arme beträgt $114 \frac{1}{2} \mathrm{~cm}$.

Das Kind macht einen geistig gut und normal entwickelten Eindruck; doch entsprechen Intelligenz und Wissen wohl nur dem wirklichen Alter des Kindes, nicht seinem Aussehen.

Der Gaumen ist spitzbogenförmig.

Figur 4.

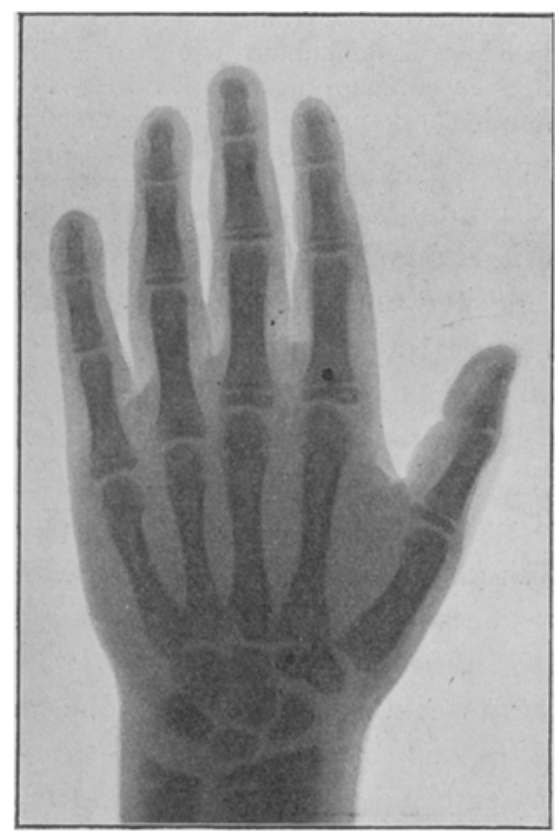

Die Schilddrüse ist fühlbar, anscheinend von der gewöhnlichen Grösse.

Der Körper des Kindes zeigt ausgesprochen runde, weibliche Formen; die Hüften sind breit. Die auffallendste Erscheinung bei der kleinen Patientin aber bilden die kräftig entwickelten, prallen Mammae, von denen jede an Volumen etwa einer Faust entspricht.

Axillarhaare sind noch nicht vorhanden.

1) Es entspricht dieses Maass etwa der.Durchschnittslänge 8-9jähriger Mädchen. Das Gewicht des Kindes ist sogar noch etwas höher. [Vgl Bendix (50).]

Archiv für Gynäkologie Bd. 94. H. 2. 
Am Mons pubis dagegen finden sich dunkele, ziemlich dichte, etwa $1 \mathrm{~cm}$ lange Haare. Die Behaarung reicht vom Mons pubis beiderseits neben der Vulva bis zu deren Mitte herab. Einzelne dunkele Haare stehen auch am Damm.

Die grossen und kleinen Labien entsprechen in ihrer Ausbildung etwa denen eines $10 \mathrm{jährigen} \mathrm{Kindes.} \mathrm{Die} \mathrm{Länge}$ der Vulva (von der Clitoris bis zum Frenulum labiorum) beträgt etwa $5 \mathrm{~cm}$. Ein Katheter lässt sich etwa $6 \mathrm{~cm}$ weit in die Vagina einführen. Der Versuch, eine vorsichtige rectale Untersuchung vorzunehmen, muss wegen der Unruhe des Kindes unterlassen werden.

Beckenmaasse: Diam. spin.: 19, Diam. crist.: $20^{1} / 2$, Diam. troch.: $23^{1} / 2$, Conj. ext.: $16 \mathrm{~cm}$.

Im übrigen macht das Kind einen durchaus gesunden Eindruck; die Untersuchung von Herz (siehe aber auch die Röntgendurchleuchtung), Lungen und Unterleib ergab nichts Besonderes.

Röntgenuntersuchung: Die Röntgenuntersuchung der rechten Hand (siehe Fig. 4) ergibt einen Knochenbau, wie er dem Alter von 10 Jahren entspricht ${ }^{1}$ ). Röntgendurchleuchtung des Thorax lässt Veränderungen an der Thymusdrüse nicht erkennen; das Herz erscheint bei der Durchleuchtung verhältnismässig gross.

Fasst man zunächst die Beobachtungen 1 bis 4 in ihrem Gesamtbilde ins Auge, so ist wohl nicht zu bezweifeln, dass es sich um eine Reihe typischer, im wesentlichen gleichartiger Fälle handelt.

Tandler und Gross (56) haben sich, im Verfolg ihrer Untersuchungen "über den Einfluss der Kastration auf den Organismus", kürzlich eingehend mit den sogenannten "Eunuchoiden" beschäftigt. Sie verstehen unter dieser Bezeichnung, nach dem Vorgange von Griffith (23) und Duckworth (15), Individuen, „deren äusserer Habitus und deren Organentwickelung dem des wahren Kastraten ähnelt" und die den gesamten Komplex der Aeusserungen aufweisen, "welche sich im Gefolge ron Genitalhypoplasie ergeben."

Auf Grund ihrer Studien an echten Eunuchen, speziell bei Skopzen, hatten Tandler und Gross zwei Typen von Eunuchen unterschieden - „den mit Disproportion im Längenwachstum und den mit extremer Fettanhäufung" --, und sie meinen, dass man in analoger Weise auch bei den Eunuchoiden diese beiden Haupttypen unterscheiden könne, "nämlich den eunuchoiden Hochwuchs, bzw. die eunuchoide Disproportion und den eunuchoiden Fettwuchs". Von Interesse ist für die vorliegende Arbeit speziell die eunuchoide Disproportion. Nach Tandler and Gross ist für diesen letzteren Typus ein gesteigertes

1) Vgl. die Abbildungen in dem Atlas von Wilms und Sick (60). 
Längenwachstum der Extremitäten charakteristisch. „Dabei trägt das Skelett als Zeichen der Unreife offene Epiphysen, jahrelang über den Zeitpunkt hinaus, zu welchem sie bei normalen Personen zu verschwinden pflegen."

Die Mitteilungen von Tandler und Gross beziehen sich hauptsächlich auf männliche Personen. Es dürfte deshalb von Interesse sein, die Resultate dieser Autoren durch Befunde zu ergänzen, die sich bei weiblichen Individuen ähnlicher Art ergeben.

Es liegt wohl nur im Sinne der Autoren, die den Ausdruck bisher gebraucht haben, wenn man die sehr prägnante Bezeichnung "Eunuchoide" auch auf weibliche Personen von ähnlicher Art anwendet, wie es die von Tandler und Gross studierten männlichen waren.

Angeregt durch die Arbeit von Tandler und Gross hat auch Peritz (47) vor kurzem bereits ein weibliches (sowie drei männliche) Individuen unter der Bezeichnung „Eunuchoide" beschrieben.

Hypoplastische Genitalien und das Fehlen wichtiger sekundärer Geschlechtscharaktere sind die Kennzeichen, die unseren Fällen in erster Linie ihr Gepräge verleihen und an das Bild von Kastraten erinnern. Ich stehe daher nicht an, die im vorhergehenden beschriebenen Patientinnen als typische Beispiele weiblicher Eunuchoide zu bezeichnen.

Dabei fand sich, in Uebereinstimmung mit den von Tandler und Gross geschilderten Merkmalen, in Fall 1,2 und 3 deutlich das Phänomen aufallend langer Extremitäten im Vergleich zur Rumpflänge, und bei allen 4 Patientinnen bestanden offene Epiphysenlinien in einem Lebensalter, wo sie normalerweise schon geschlossen sein müssten.

(Fettwuchs dagegen war nie vorhanden. Im Fall 4 bestand eine nur geringe Adipositas, in Fall 1 und 3 sogar auffallende Magerkeit.)

Die Beobachtungen sind also, wenn man der Einteilung von Tandler und Gross folgt, unter den Typus der eunuchoiden Disproportion zu zählen.

Tandler und Gross (56) haben nun versucht, zwischen dem Typus der Eunuchoide and dem des Infantilismuseine scharfe Grenze zu ziehen. Sie sagen, dass die Disproportion, die der Körper vieler Eunuchoide zeigt, diesen „Typus vom infantilen trennt, sofern man unter Infantilismus das Erhaltensein der kindlichen Körperproportionen versteht ${ }^{4} .1$ )

1) \#Bei dem Fötus und dem kleinen Kinde überwiegt die Länge des Ruınpfes, während später während der Pubertät die Extremitäten stärker wachsen" [A. Hegar (31)]. 
Diese Abgrenzung beruht indessen auf einer so eng gefassten Definition des Begriffes "Infantilismus", wie ich sie nicht für berechtigt halte. Meiner Meinung nach muss man sämtliche Krankheitsformen in das Gebiet des Infantilismus (bzw. Fötalismus) zählen, bei denen überhaupt als wesentliches Moment eine Hemmungsbildung eine Rolle spielt; vermöge deren ein oder mehrere Organe des Körpers auf kindlicher (bzw. fötaler) Stufe der Entwickelungstehen geblieben sind. Welche und wieviele Organe des Körpers. diese Hemmungsbildung zeigen, ist für die allgemeine Definition des Begriffes "Infantilismus" unwesentlich und kann nur für eine speziellere Einteilung in Betracht kommen. So ist auch das Erhaltensein der kindlichen Körperproportionen, auf das Tandler und Gross einen so besonderen Wert legen, meiner Ansicht nach, nur als eine der vielen Erscheinungsmöglichkeiten des. Infantilismus anzusehen.

Wenn von manchen Autoren eine Einteilung in universellen Infantilismus und partiellen befolgt wird, [vgl. Fromme (22)], so ist in praxi dagegen nichts einzuwenden; nur muss man dabei im Auge behalten, dass man im strengen Sinne des Wortes von universellem Infantilismus nur dann reden dürfte, wenn ein Individuum wirklich in allen seinen körperlichen und geistigen Funktionen auf einer kindlichen Stufe zurückbleibt.

Ob im strengen Sinne des Wortes ein universeller Infantilismus überhaupt vorkommt, ist mindestens zweifelhaft. Wahrscheinlich ist jeder Fall von Infantilismus nur ein partieller, wenn auch von sehr verschiedener Ausdehnung.

Daraus folgt, dass in der Entwickelung der Körperteile infantiler Individuen wohl immer eine Inkongruenz eintreten muss, dergestalt, dass weder ganz die normalen Proportionen des Kindes. noch die normalen Proportionen des Erwachsenen sich herausbilden. Disproportion wird damit geradezu zu einer charakteristischen Begleiterscheinung des Infantilismus, und eben in dieser Inkongruenz im Wachstum dürfte - das ist der praktisch wichtige Punkt - ein Teil der Schädlichkeiten begründet. sein, die dem Infantilismus anhaften.

Ebenso wie unter den anderen Missbildungen heben sich nun auch unter den infantilen einige besonders häufige oder besonders. eigenartige Formen als Typen hervor, und - sofern die Genitalhypoplasie bei Eunuchoiden auf einer infantilen Hemmungsbildung beruht, - stellen solche Individuen nur 
einen eigenartigen und dabei besonders interessanten Typus des Infantilismus dar.

Dass es sich bei unseren Fällen in diesem Sinne um infantilistische Eunuchoide gehandelt hat, werde ich in folgendem noch zu beweisen suchen.

Da der Infantilismus vom allgemein-pathologischen Standpunkte aus als eine echte Missbildung aufzufassen ist, ${ }^{1}$ ) so wollen wir uns auch an Begriffe halten, die in der Teratologie speziell üblich sind, und bei der weiteren Besprechung unserer Fälle die Frage stellen nach ihrer formalen und nach ihrer causalen Genese.

Dabei sei kurz bemerkt, dass man unter dér formalen Genese die Entwickelungsgeschichte der Terata versteht; die formale Genese betrifft die Frage danach, welche von den verschiedenen Missbildungen, die ein Monstrum aufweist, die primäre war, ferner die Fragenach dem Abhängigkeitsverh ältnis der einzelnen Entwickelungsanomalien des Monstrums von einander oder nach ihrer gemeinsamen Ursache. Die kausale Genese dagegen betrifft die Frage nach den Ursachen, welche die Gesamtheit der abweichenden Vorgänge bei einer Missgeburt bedingten. [Vgl. hierzu E. Schwalbe's (49) Auseinandersetzungen in seinem grundlegenden Werke, dem diese Definitionen entnommen sind.]

Die Frage nach der formalen Genese der infantilistischen Eunuchoide würde nun viel leichter, als dies tatsächlich der Fall ist, zu beantworten sein, wenn man die in Rede stehenden Individuen in vollem Masse in Parallele zu solchen stellen könnte, die in der Jugend kastriert wurden; denn dann dürfte man die Resultate, die sich aus Beobachtungen an kastrierten Menschen und Tieren ergeben, ohne weiteres zur Deutung der Befunde bei den Eunuchoiden verwerten.

So einfach aber liegen die Dinge nicht, wie sich aus folgendem ergibt:

Uebereinstimmend zeigen fast alle Erfahrungen, die darüber vorliegen, dass in der. Jugend kastrierte, vorher

1) Aus praktischen Gründen kann man allerdings, wie E. Schwalbe (49) auseinandersetzt, vorziehen, den Begriff einer "Missbildung" nur auf Störungen der embryonalen Entwickelung zu beschränken und nicht auf solche der postembryonalen, wie es der Infantilismus ist, auszudehnen. Im Prinzip sind aber sicherlich beide von den gleichen Gesichtspunkten aus aufzufassen. Es kommt hinzu, dass man klinisch nichtimmer imstande ist, zwischen Infantilismus and Fötalismus genau zu unterscheiden. 
gesunde Menschen sowohl wie Tiere an Körperlänge und Gewicht über das gewöhnliche Maass hinaus wachsen.

Im einzelnen sei hierzu folgendes kurz erwähnt:

Ueber die Folgen der Kastration männlicher Kinder ist man ziemlich gut unterrichtet:

Bilharz [citiert nach Sellheim (51)] erzählt, dass die Eunuchen, die er gesehen hat, lauter ungewöhnlich lange Gestalten von übrigens schlechter Haltung gewesen seien.

Merschejewsky [citiert nach Tandler und Gross (55)] führt. an, dass nach ,seinen an 22 Skopzen im Alter von 25-73 Jahren erhobenen Mittelmaassen die Körperlänge der Skopzen $169 \mathrm{~cm}$ betrage, |während die durchsehnittliche Körperlänge der Nichtkastrierten $165,6 \mathrm{~cm}$ betrage."

Noch grössere Maasse fanden Tandler und Gross (54a): Sie sagen: ,Von den 4 Frühkastrierten, die wir untersuchten, war der kleinste $173,5 \mathrm{~cm}$, der grösste $190 \mathrm{~cm}$ lang. Das relativ geringe Durchschnittsmaass der Skopzen, zu dem Merschejewsky gelangt ist, dürfte zum Teil darauf zurückzuführen sein, dass er Spätkastraten von Frühkastraten nicht geschieden hat." (Dass Tandler und Gross. neben dem Haupttypus der hochgewachsenen Ennuchen den mit Fettwuchs unterscheiden, wurde schon oben erwähnt.)

Was die Folgen der Kastration weiblicher Kinder anbetrifft, so wissen wir darüber bisher allerdings ausserordentlich wenig. Ich finde nur eine Angabe von Roberts, die Hegar (27) schon 1878 erwähnt, auch in den neueren Arbeiten immer wieder zitiert. Roberts will auf einer Reise in Indien weibliche Kastraten gesehen haben, die von ihm als "gross, muskulös und vollkommen gesund" geschildert werden. [Zitiert nach Hegar (27)].

Ueber die Folgen der Kastration bei Tieren schliesslich liegen zuverlässige Experimente neuerer Forscher vor. Wie Sellheim (51) gezeigt hat, fällt die Zunahme an Gewicht und Körperlänge besonders ins Ange. Beim Rinde ist nach Tandler und Keller (57) "das auffallendste in der äusseren Erscheinung der weiblichen Kastraten", "die bedeutende Höhe dieser Tiere."

Demgegenüber ist es Sellheim (51) schon aufgefallen, dass die "sogenannten natürlichen Eunuchen, Individuen mit kongenitalem Defekt der Hoden, bei welchen man vielleicht ein gesteigertes Wachstum des Körpers erwarten dürfte", nach einer Zusammenstellung, die Gruber (24) im Jahre 1868 geliefert hat, „im Gegenteil von schwächlichem Bau" sind. „Doch müssen wir," sagt Sellheim, "bedenken, das die einsehlägigen Beobachtungen sehr spärlich und lückenhaft sind. "1)

Auch bei den von uns beobachteten infantilen weiblichen Eunuchoiden zeigt sich nun in der Tat, dass alle diese

1) Tandler und Gross (56) kommen zu dem Resultat, dass die von Gruber gesammelten Fälle von beiderseitiger Anorchie einer wissenschaftlichen. Kritik nicht standhalten. 
4 Patientinnen von'kleiner, zum Teil sogar von sehr kleiner Statur waren. Dabei waren 2 von ihnen sehr mager; alle waren von schwächlicher, äusserst dürftig und hinfällig erscheinender Konstitution.

Auch der von Peritz (47) kürzlich beschriebene weibliche Eunuchoid war „mager und grazil gebaut und nicht über das Mittelmaass gross."

Unter ihren männlichen Eunuch oiden haben allerdings Tandler und Gross (56) auch solche mit Hochwuchs und Fettwuchs beobachtet, doch bemerken auch sie: „In anderen Fällen ist die Gesamtlänge des Individuums trotz des gesteigerten Wachstum eine das Durchschnittsmaass nicht übertreffende, ja, sie kann unter diesem bleiben."

Danach scheint mir ein wesentlicher Unterschied zwischen der Entwickelung der Eunuchen und der Eunuchoide im grossen und ganzen (oder mindestens einer Gruppe von Eunuchoiden, zu der unsere Fälle gehören) unverkennbar. Auf eine weitere Verschiedenheit zwischen Eunuchen und Eunuchoiden werden wir unten (S. 570) noch zu sprechen kommen.

Die Differenz hinsichtlich der Körpergrösse ist um so bemerkenswerter, als gerade dasjenige $\mathrm{Pbänomen,} \mathrm{mit} \mathrm{dem} \mathrm{das} \mathrm{ge-}$ steigerte Längenwachstum kastrierter Menschen und Tiere wohl zweifellos zusammenhängt - nämlich das Offenbleiben der Epiphysenlinien — sich auch bei den Eunuchoiden findet, wie unsere sämtlichen 4 Beobachtungen zeigen. Wenn diese Personen im Gegensatz zu der Mehrzahl der Kastraten trotzdem so klein bleiben, so müssen dabei irgendwelche Momente eine Rolle spielen, die bei den Kastraten nicht vorliegen. Es handelt sich hier eben nicht um natürliche Eunuchen", wie Sellheim (51) und Halban (25) Personen mit angeborenem Defekt der Keimdrüsen nennen, sondern um Individuen, die man gerade deshalb nur als „Eunuchoide ${ }^{\text {. }}$ bezeichnen soll, weil bei ihnen die mangelhafte Funktion der Keimdrüsen lediglich eine Teilerscheinung einer allgemeinen Erkrankung oder Missbildung 'ist. ${ }^{1}$ )

1) Man kann die Frage aufwerfen, die auch Tandler und Gross (56) berühren, inwieweit zwischen der Krankheitsform der Eunuchoide und der sogenannten lymphatischen Konstitution, wie sie Paltauf geschildert, oder der "hypoplastischen Konstitution", wie sie an der Hand von Sektionsmaterial Bartel (2) beschrieben hat, oder ähnlichen Konstitutionsnomalien [astbenische Konstitutionskrankbeit - Stiller (52)] eine Verwandtschaft besteht. Nach Bartel ist Hypoplasie des Genitalapparates mit Uterus infantilis beim Weibe als eine Teilerscheinung der hypoplastischen Konstitution anzusehen. Auffallend ist aber, dass nach E. Herr- 
Es ist mit dem Infantilismus wahrscheinlich überhaupt nicht anders als mit dem ihm in mancher Hinsicht nahe verwandten Hermaphroditismus. Wie Virchow (58) und Neugebauer (45) gezeigt haben, ist auch der Hermaphroditismus "keine lokalisierte Missbildung der Genitalien", sondern "eine Teilerscheinung einer allgemeinen Degeneration, sehr häufig vergesellschaftet mit anderen Missbildungen".

Daraus folgt aber, dass man nur mit grösster Vorsicht experimentelle Erfahrungen auf die Eunuchoide übertragen und vorläufig immer nur mit einem mehr oder weniger hohen Grade von Wahrscheinlichkeit die Frage beantworten kann, welche von den vorhandenen Anomalien bei den Eunuchoiden primäre Missbildungen und welche erst wieder Folgeerscheinungen der Genitalhypoplasie sind. -

Die Befunde, die wir bei unseren Fällen im einzelnen zu erörtern haben, sind: Anomalien an den Genitalien, Anomalien in der Ausbildung der sekundären Geschlechtscharaktere, an innersecretorischen Drüsen und in der Entwickelung des Skeletsystems.

Was zunächst den Genitalapparat anbetrifft, so waren in den sämtlichen 4 Beobachtungen die äusseren Genitalien sowohl wie der Damm durchaus normal gebildet; Darm- und Harnapparat mündeten an der gewöhnlichen Stelle, und alle diese Teile böten kein auffallendes Bild, wenn sie einem Kinde angehört hätten. Ganz dasselbe gilt von der Scheide; in Fall 1, 2 und 3 war eine mehr oder weniger enge, den Verhältnissen beim Kinde durchaus entsprechende Scheide von gewöhnlicher Länge vorhanden.

In Fall 4 wurde die Länge der Scheide nicht gemessen, doch bestanden hier in dieser Hinsicht zweifellos normale oder annähernd normale Verhältnisse.

Der Uterus konnte in einer Beobachtung (Fall 4) als kleiner

mann (34) bei der hypoplastischen Konstitution (Bartel) die Ovarien in 58 pCt. der Fälle vergrössert gefunden wurden, während der Uterus meist hypoplastisch war. Darin scheint mir unter allen Umständen eine wesentliche Differenz gegenüber unseren Beobachtungen zu liegen, in denen von einer Vergrösserung der Ovarien, soweit die klinische Untersuchung irgend ein Urteil erlaubt, wohl keine Rede sein kann [siehe auch die Arbeit von Bartel und Herrmann (3)]. 
Körper mit Sicherheit, in 2 Beobachtungen (Fall 1 und 2) mit einiger Wahrscheinlichkeit nachgewiesen werden.

In Fall 3 war wegen der Enge auch des Anus und Mastdarms die rectale Untersuchung so erschwert, dass kein Urteil über den inneren Genitalbefund möglich war.

Was die Tuben und die Ovarien anbetrifft, so war es allerdings nicht möglich, diese Organe palpatorisch nachzuweisen. Bei der Seltenheit eines wirklichen Defektes der Keimdrüsen ist aber immerhin wahrscheinlich, dass hypoplastische Keimdrüsen (siehe unten Seite 565) vorhanden gewesen sind.

Irgendwelche anderweitigen Missbildungen, wie Doppelbildungen oder ähnliches, waren nicht zu konstatieren.

Nach alledem glaube ich, dass die Diagnose wohl mit Recht auf eine infantile Hemmungsbildung am Genitalapparat gestellt werden durfte. (Auf den feineren Unterschied zwischen Fötalismus und Infantilismus soll dabei allerdings mangels eines Inspektionsbefundes kein Wert gelegt werden.)

Wir nehmen damit an, dass in diesen Fällen die Müllerschen Fäden beim Embryo angelegt waren und dass die allerersten Entwickelungsstadien dieser Fäden obne nachweisbare Abweichungen vor sich gegangen sind, dass alsdann aber noch im fötalen oder im kindlichen Alter ein Stillstand in der Entwickelung der aus diesen Fäden normalerweise hervorgehenden Organe eingetreten ist.

Man muss sich fragen, ob dieser Entwickelungsstillstand nur die Folgeerscheinung einer Entwickelungsstörung der Keimdrüsen gewesen ist oder ob es sich dabei um eine primäre Anomalie der Eunuchoide handelte; denn die Schädigung, die die Ursache der ganzen Krankheitsform war, könnte ja auch direkt nicht nur die Keimdrüsen, sondern auch andere Teile des Genitalapparates in ihrem Wachstum beeinträchtigt haben.

A. Hegar (29) hat in der Tat gewichtige Gründe dafür angeführt, dass ein unbedingter Kausalnexus zwischen solchen Defekten nicht besteht.

Mit Recht sagt auch Halban (25), der einen ähnlichen Punkt berührt: „Wir dürfen aus dem negativen Befunde (Defekt der Keimdrüse und rudimentäre Entwickelung des Penis z. B.) nicht den Schluss ziehen, dass das eine die Konsequenz des anderen sei; es besteht vielmehr die Möglichkeit, dass beide Entwickelungsstőrungen auf eine dritte Ursache zurückzuführen sind. Aehnliche Betrachtungen ergeben sich auch bei bilateraler Agenesie der Ovarien." 
Immerhin wird eine Entscheidung dieser Frage erst auf Grund weiterer Beobachtungen möglich sein. Man kann vorläufig nur sagen, dass nach den Erfahrungen, die man bei frühzeitig Kastrierten gemacht hat, bei der fehlenden Funktion der Keimdrüsen die übrigen Genitalorgane zu einer vollkommenen Ausbildung jedenfalls nicht gelangen konnten. -

Es gibt nun Missbildungen, die eine gewisse Aehnlichkeit mit der infantilen Beschaffenheit des Genitalapparates der hier beschriebenen Eunuchoide aufweisen und daher einen Vergleich mit diesen herausfordern. In erster Reihe kommt dabei der vollständige Mangel und die sogenannte rudimentäre Anlage der Müllerschen Fäden in Betracht. ${ }^{1}$ ) Wie es scheint, haben Fälle der letzteren Art in der gynäkologischen Literatur bisher eine viel grössere Beachtung gefunden, als die Eunuchoide.

Im Vergleich zur infantilen Entwickelung ist die rudimentäre Anlage der Müllerschen Fäden natürlich die embryologisch frühzeitigere und viel hochgradigere Missbildung. Dementsprechend finden wir die charakteristische Erscheinung, dass bei der rudimentären Anlage -, mag sich dabei ein Uterus rudimentarius partim excavatus oder ein Uterus rudimentarius solidus finden - , die Scheide meistens entweder ganz obliteriert oder nur in ihrem unteren Abschnitt als kurzes Hohlorgan nachweisbar ist [zitiert nach Menge (43)].

Trotz dieser hochgradigeren Missbildung am Genitalapparat aber zeigt sich auffallenderweise, dass die Trägerinnen dieser Anomalie als Gesamtindividuen gewöhnlich viel besser ausgebildet sind, als die infantilen. Im Gegensatz zu den Eunuchoiden sind die sogenannten sekundären Geschlechtscharaktere in den Fällen, wo die Scheide obliteriert ist, meistens ganz normal ausgeprägt.

Mehrfach wird auch berichtet, dass derartige Personen den Wunsch hatten, zu heiraten, und bekanntlich hat man in solchen

1) Nach v. Winckel (61), der 7 Entwickelungsstufen der Müllerschen Fäden unterscheidet, entspricht: der vollständige Mangel beider Fäden der I. Stufe (1.-4. Embryonalwoche); der Uterus rudimentarius der II. Stufe (4.-8. Embryonalwoche); die Ausgestaltung des Uterus foetal is der V. Stufe (20.-40. Schwangerschaftswoche); der Uterus infantilis der VI. Stufe (1.-10. Lebensjahr). 
Fällen zuweilen, um Kohabitationen zu ermöglichen, eine Scheide operativ zu bilden versucht. ${ }^{1}$ )

Menge (43) sagt bei Besprechung des Uterus rudimentarius: „Brüste, Achselhaare, Schamhaare verhalten sich, ebenso wie die äusseren Genitalien, vorwiegend normal. Nagel gibt an, dass die Stimme und das Benehmen der hierher gehörigen Individuen weiblich seien. Neigung zum männlichen Geschlecht sei gewöhnlich vorhanden, mitunter in gesteigertem Maasse".

Diese Tatsachen sind um so merkwürdiger, als bei einzelnen Personen mit fehlender Scheide und Gebärmutter die genauere Untersuchung ergab, dass sie trotz ihrer sekundären weiblichen Sexualcharaktere gar keine Weiber waren, sondern dass die vorhandenen Keimdrüsen Hoden gewesen sind [siehe die Arbeit von Heyn (34a) and Beobachtungen von Blumreich ${ }^{2}$ ) (8) und Helmbold (32)].

1) Um ein Beispiel anzuführen, sei auf einen sehr typischen Fall hier hingewiesen, den Menge (43) beobachtete: 21jähriges Mädchen. „Die Patientin ist verlobt und hat den dringenden Wunsch, sich bald zu verheiraten. Sie hat auch wiederholt Kobabitationsversuche gemacht. Dabei hat es sich herausgestellt, dass eine perfekte Kohabitation unmöglich ist, dass aber Libido und Voluptas ausgesprochen vorhanden sind. Untersuchungsbefund: Gesundes, blühend aussehendes Mädchen von sehr kräftigem Körperbau, aber durchaus weiblichem Habitus. Brüste halbkugelig aufsitzend, mittelgross. Drüsenparenchym gut entwickelt; reichliche Achselbaare, weibliche Beckenform. Vulva und Vestibulum völlig normal gebildet. Auf dem Mons veneris und an den grossen Labien dichte Behaarung. Clitoris gut entwickelt. Urethralmündung etwas geweitet, so dass man die Fingerkuppe in sie einlegen kann. Die tieferen $\Delta b$. schnitte der Urethra sind dagegen eng. Unterhalb der Harnröhrenmündung ein gelappter kräftiger Hymen denticulatus. Der Scheideneingang ist für einen Finger gut durchgängig und führt in einen ganz kurzen häntigen Blindsack, der ea. $2 \mathrm{~cm}$ tief ist und sich trichterförmig etwas verjüngt. Das kurze gehöhlte Scheidenrudiment ist aber gut dehnbar, so dass man den Finger ungefähr $4 \mathrm{~cm}$ tief in den Blindsack einpressen kann." "Es gelingt nicht, irgendwelche Gewebspartien zu fühlen, die man als Rudimente der oberen Vaginalteile oder des Uterushalses ansprechen könnte. " Dagegen ist ein bogenförmiger, fast bleistiftdicker Strang zu fühlen, von dem anzunehmen ist, dass es sich um ein bogenförmiges Uterusrudiment handelt. Anzunehmen ist ferner, naeb dem Befunde, dass die unteren Abschnitte der Müllerschen Fäden völlig fehlen oder dass sie nur so kümmerlich entwickelt sind, dass sie sich dem Tastnachweis völlig entziehen. Zwei seitlich liegende, fühlbare Körper ${ }_{1}$ sind unzweifelhaft die etwas hypoplastischen Ovarien, in denen aber nach der Anamnese, gut funktionierendes Ovarialgewebe vorhanden sein muss." [Die Abbildung eines ähnlichen Falles siehe in der Arbeit von B ättner (12)].

2) In dem Fall Blumreich handelte es sich um eine Person von 22 Jahren. „Man konnte von Uterus und Ovarien klinisch nichts Deutliches nach- 
Zwischen den infantilen Eunuchoiden und den Individuen mit rudimentärer Anlage der Müllerschen Fäden bestehen danach nicht nur hinsichtlich der zeitlichen Entwickelung und der Morphologie der Genitalmissbildung wesentliche Differenzen. Der ganz verschiedene Habitus dieser Personen beweist vielmehr, dass diese beiden Gruppen auch allgemein biologisch und ätiologisch ganz verschjeden aufzufassen sind. Dieser Tatsache, auf die kürzlich auch Menge (43) and Holzbach (36) hingewiesen haben, ist nicht immer genügend Rechnung getragen worden. Dass Analogieschlüsse, die zwischen Infantilismus und rudimentärer Anlage der Müllerschen Fäden gezogen wurden, nicht berechtigt sind, ist von Menge (43) treffend hervorgehoben worden.

Die Entwickelungshemmungen der allerfrühesten Zeit des Embryonallebens, wie man sie als Embryonalismus bezeichnet hat, dürften überhaupt als etwas vom Fötalismus oder gar Infantilismus prinzipiell Verschiedenes anzusehen sein.

Natürlich steht es damit nicht im Widerspruch, wenn, wie überall in der Teratologie, so auch hier, zuweilen $\mathrm{Kombinationen}$ auftreten, und in einzelnen Fällen ein eunuchoider Typus mit rudimentärer Anlage der Müllerschen Fäden vergesellschaftet gefunden wird.

weisen, die Scheide bildete einen kurzen Blindsack, die äusseren Genitalien zeigten keinerlei Abnormität; es lag ausgesprochen weiblicher Habitus und weibliches Empfinden, Hinneigung zu Männern vor. Brüste, Becken, Körperformen, Antlitz, Stimme absolut weiblich." In beiden Leisten dagegen fand sich ein kirschgrosser Körper, der sich hart anfühlte. Der linksseitige Körper wurde, da er schmerzte, herausgeschnitten und die mikroskopische Untersuchung duroh Geheimrat Waldeyer ergab, dass es sich um einen auf fötaler Entwickelungsstufe stehengebliebenen Hoden handelte. Za diesem Falle bemerkte in der Diskussion Hocheisen (35), dass bei der Patientin später wegen Schmerzen auch auf der rechten Seite und weil eine sarkomatöse Entartung des Leistenhodens zu befürchten war, anch der in - der rechten Leistengegend befindliche Körper entfernt wurde. „Es war mikroskopisch deutlich ein Hoden. Der Erfolg der Operation war aber der, dass das Mädchen eine sehx schwere Psychose bekam, an der sie jetzt 2Jahre schon leidet." Ho ch e i s en warnt aus diesem Grunde davor, „zu sehr mit der Operation in einem solchen Falle vorzugehen." - Dieser Verlauf zeigt, meines Erachtens, dass hier wohl zweifellos funktionierendes Hodengewebe vorhanden gewesen ist, wenn auch wahrscheinlich nicht im Sinne einer Produktion von Keimzellen, so doch im Sinne einer inneren Sekretion (Hormonwirkung). 
(Burrage (11) fand unter 35 Sektionsfällen von rudimentärer Entwickelung der Müllerschen Fäden $6 \mathrm{mal} \mathrm{Fehlen} \mathrm{der} \mathrm{0varien.)}$

Einen Fall, in dem vermutlich eine Kombination von eunuchoidem Typus mit rudimentärer Bildung der Müllerschen Fäden vorlag, hat Bunge (10) mitgeteilt: Es handelte sich um ein 22 Jahre altes, nie menstruiertes Mädchen. Weder Uterus noch Ovarien waren festzustellen. Hinter dem Hymenalrest fand sich an Stelle der Scheide nur ein Blindsack, der durch Dehnung mittels Specula allmählich so weit wurde, dass man mit dem Daumenglied gerade hineingelangen konnte. Die grossen Labien waren leidlich ausgebildet, die kleinen Labien vorhanden, die Schamhaare mangelhaft, die Brüste schwach entwickelt, sexuelles Empfinden war vorhanden.

Gelegentlich scheint auch die Kombination eines eunuchoiden weiblichen Habitus mit hypoplastischen Hoden vorzukommen.

Ein Fall, den P. Strassmann (53) berichtet hat, könnte vielleicht in diesem Sinne aufzufassen sein:

P. Strassmanns Patientin war 22 Jahre alt. Die änsseren Genitalien waren durchaus weiblich gebildet, die Scheide geräumig, blind endigend, vom Uterus nichts zu fühlen. Eunuchoiden Typus könnte man hier darin erblicken, dass bei der Patientin die Behaarung am Mons pubis auffallend gering war und die Brüste ,in der Mitte zwischen Mann und Weib" standen. In den Leistengegenden fühlte man sich vordrängende höckerige Körperchen. Ein excidiertes Stückchen ergab Ho dengewebe.

Derartige Beobachtungen sind aber sicherlich nur A usnahmen, und, wenn wir auch in unseren Fällen die Keimdrüsen nicht direkt untersuchen konnten, so ist doch jedenfalls nicht der geringste Grund vorhanden, anzunehmen, dass etwa auch bei unseren Patienten die Keimdrüsen hypoplastische $\mathrm{Hoden}$ gewesen seien ${ }^{1}$ ).

Eher noch könnte man daran denken, dass die Keimdrüsen hier vielleicht überhaupt keinen anatomisch ausgesprochenen Geschlechtscharakter hatten, wie dies nach R. Virchow (59) und L. Pick $(48)^{2}$ ) zuweilen vorkommen soll. -

Was nun die Ausbildung oder das Fehlen der sekundären Geschlechtscharaktere bei den Eunuchoiden anbetrifft, so scheint mir sehr bemerkenswert, dass unsere sümtlichen 4 Fälle ziemlich deutlich diejenigen sekundären Sexualcharaktere aufwiesen, die das

1) Im Speziellen waren in keinem der Fälle Erscheinungen vorhanden; die auf einen Leistenhoden hätten hinweisen können, wie er sich beim Pseudohermaphroditismus, nicht selten findet:

2) Pick sagt: "Neben den echten Zwittern mit männlicher und weiblicher Geschlechtsdrüse und den Scheinzwittern (Pseudohermaphroditen), die entweder geschlechtlich missbildete Männer oder geschlechtlich missbildete Weiber mit geschlechtssicheren Keimdrüsen sind, gibt es Individuen neutrius generis." 
weibliche Kind vom männlichen unterscheiden. Besonders in psychischer Hinsicht war der mädchenhafte Charakter der Patientinnen stets ganz unverkennbar. Auch das lange und volle Kopfhaar dürfte wohl in diesem Sinne als ein feminines Zeichen anzuführen sein [vgl. Halban (25) und Bayer (4)] ${ }^{1}$ ).

Es fehlten also den infantilen Eunuchoiden typischerweise von den weiblichen Geschlechtscharakteren nur solche, die sich erst in der Pubertät ausbilden, und zwar in Fall 1, 2 und 3 so gut wie vollständig, die Schamhaare und Achselhaare, die weiblichen Brüste und Mammillen, die Entwickelung der weiblichen Beckenund allgemeinen weiblichen Körperformen und wahrscheinlich auch die Libido sexualis. In Fall 4 waren einige dieser Symptome zwar angedeutet, aber nur in sehr geringem Maasse.

Auch hier ist wieder die Frage berechtigt, ob die Defekte Folgeerscheinungen der Keimdrüsenhypoplasie oder primäre Anomalien solcher Individuen sind.

Dass bei Kastraten nach frühzeitiger Exstirpation der Keimdrüsen die sekundären Geschlechtscharaktere in ähnlicher Weise wie hier unentwickelt bleiben, ist sichergestellt. Beobachtungen von Tandler und Gross bei männlichen Eunuchoiden ferner zeigen, dass auch diesen Personen die für ihr Geschlecht charakteristischen Merkmale, soweit sie sich in der Pubertät entwickeln, fehlen.

$\mathrm{Da}$ eine solche Uebereinstimmung besteht, so ist es bis zu einem gewissen Grade wahrscheinlich, wenn auch keineswegs absolut sicher, dass der Defekt der in Rede stehenden Sexualcharaktere eine direkte Folgeerscheinung der Keimdrüsenhypoplasie ist.

Von besonderer Wichtigkeit ist folgendes:

Bis in die neueste Zeit hinein besteht vielfach die Ansicht, dass der Ausfall der Funktion der Eierstöcke imstande sei, bei Frauen männliche Geschlechtscharaktere geradezu hervortreten zu lassen. Es wird berichtet, dass Frauen nach der Kastration oder nach dem. Klimakterium eine männliche Stimme oder Bartbildung bekommen hätten. (Andererseits soll bei Männern nach der Atrophie oder Exstirpation der Hoden Gynäkomastie entstanden sein).

1) Bayer (4) schreibt: „Die Kopfhaare sind im weiblichen Geschlecht reichlicher und länger, meist auch dicker, eine Erscheinung, die bereits im Kindesalter zu konstatieren ist." " 
Im Zusammenhang hiermit steht die besonders von Herbst (33) vertretene Ansicht, dass die funktionierenden Keimdrüsen nicht nur eine positive, sondern auch eine negative Wirkung ausüben: sie sollen, verhindern, dass die sekundären Sexualcharaktere des entgegengesetzten Geschlechts in die Erscheinung treten" (Herbst).

Biedl (7) sagt in seinem kürzlich erschienenen Werke „Ueber die innere Sekretion": "Nicht selten sind die Beobachtungen, in welchen ein mehr oder weniger vollständiger Schwund einzelner ausgeprägter Merkmale des einen Geschlechts und das Auftreten solcher des anderen verzeichnet werden. Zumeist handelt es sich um traumatisch ausgelöste oder idiopathische pathologische Prozesse an den Keimdrüsen, nach welchen eine solche Veränderung der Sexualcharaktere eingetreten ist." "So sieht man in der Menopause nicht gar selten das Auftreten von virilen Charakteren, eine tiefe männliche Stimme, mehr oder weniger starke Bartentwickelung und Behaarung des Körpers". (Siehe hierzu S. 568 Anm.)

Hegar (28), Alterthum (1) und Halban (25) haben derartige Behauptungen schon zu entkräften und zu zeigen versucht, dass es sich dabei teils um Beobachtungsfehler, teils um Beobachtungen an Personen gehandelt hat, die schon von vornherein heteros exuelle Charaktere aufwiesen, wie dies besonders hinsichtlich des Haarkleides bei Frauen keine Seltenheit ist. Tandler und Gross meinen ausserdem, dass manche Symptome, die gewöhnlich als Charaktere nur des einen Geschlechtes gedeutet werden, in Wirklichkeit allgemeine Artcharaktere des Menschen sind. Franz (18) zeigte im speziellen, dass nach Wegnahme der Geschlechtsdrüsen im jugendlichen Alter bei den Versuchstieren sich Becken ausbilden, die zwischen männlichen und weiblichen Becken die Mitte halten und nur ganz schwache Geschlechtscharaktere zeigen. Der Beckentypus des männlichen Tieres tritt nach der Kastration weiblicher Tiere also nicht auf.

Unsere Beobachtungen liefern für die Ansicht der soeben genannten Autoren eine wesentliche Stütze; denn trotz der hier von Jugend an wohl zweifellos bestehenden Hypoplasie der Keimdrïsen, hat sich bei diesen 4 Personen kein einziges viriles Merkmal entwickelt.

Nun ist aber bekannt, dass man bei Frauen mit geringeren Graden von Infantilismus am Genitalapparat, wo die Menstruation besteht, die Eierstöcke also funktionieren, gar nicht selten eine virile Art des Knochenbaus oder den Typus der virilen Behaarung beobachtet. A. Mayer (41) 
und E. Kehrer (38) haben auf solche Befunde neuerdings besonders aufmerksam gemacht.

Sind also virile Merkmale an sich gar nicht selten mit Infantilismus bei Frauen kombiniert, so könnte man solche heterosexuellen Symptome doch dann erst recht bei den höchsten Graden des Infantilismus, also bei den Eunuchoiden, erwarten. Wenn sie sich, wie wir sehen, trotzdem gerade bei diesen böchsten Graden infantiler Missbildungen nicht zeigen, so erklärt sich das vielleicht so, dass sie auch hier in der Anlage zuweilen vorhanden sind, aber nicht zur Ausbildung gelangten, also nicht in Erscheinung treten konnten, weil eben bei Eunuchoiden die Funktion der Keimdrüsen und damit jeder Impuls zur Entwickelung irgendwelcher Geschlechtscharaktere in der Pubertätszeit gefehlt hat.

Damit würden wir auf den Standpunkt einer Theorie gelangen, die besonders von Halban (25) neuerdings ausgebaut ist. Die Anlagen der einzelnen Geschlechtscharaktere sind nach dieser Theorie von den Keimdrüsen ganz unabhängig. Die Keimdrüsen besitzen jedoch in der Pubertätszeit auf die volle $\Lambda$ usbildung der Sexualcharaktere einen "protektiven Einfluss". Dabei genügt es aber für die Entfaltung der sekundären Geschlechtscharaktere, dass überhaupt eine funktionierende Keimdrüse im Körper vorhanden ist, gleichgültig ob jene Charaktere im selben Sinne wie die betreffende Keimdrüse oder - bei Missbildungen - heterosexuell angelegt sind.

Gegen die allgemeine biologische Giltigkeit dieser Theorie lassen sich zwar aus experimentellen und zoologischen Gründen noch Einwendungen erheben [rgl. Bresea (9) und Biedl (7) $\left.{ }^{1}\right)$;

1) Nach der Theorie von Biedl (7) ist die Anlage der Keimdrüsen normalerweise stets eine bisexuelle, echthernaphroditische. Je nachdem der männliche oder weibliche Teil der Keimdrüsen das Uebergewicht gewinnt, gelangen auch die entsprechenden Sexualcharaktere bei dem Individuum, unter dem Einfluss des von der Keimdrüse gelieferten spezifischen Hormons zur Ausbildung. Wenn non bei Frauen die Eierstöoke ibre Funktion im Klimakterium einstellen, oder wenn diese Organe "durch traumatisch ausgelöste oder idiopathische, pathologische Prozesse" zerstört werden, so kann der übrigbleibende rudimentäre, heterosexuelle Anteil der Keimdrüsen das Uebergewicht. gewinnen und nun virile Charaktere bei Franen zur Ausbildung kommen lassen (siehe oben Seite 566, 567). - Ich möchte gegen diese Hypothese folgendes einwenden: Wenn traumatisoh ausgelöste oder idiopathische pathologische Prozesse die Keimdrüsen zerstören, so ist doch kaum an- 
sie ist, meiner Ansicht nach, aber bisher am besten imstande, die Erfahrungen wenigstens der menschlichen Pathologie verständlich zu machen.

Jedenfalls sind die Befunde beim Infantilismus mit der Halbanschen Annahme vorläufig gut in Einklang zu bringen und daher geeignet, ihr eine Stütze zu geben. -

Wir kommen jetzt zur Besprechung der Drüsen mit innerer Sekretion bei den Eunuchoiden:

Durch eine grosse Reihe von Arbeiten ist sichergestellt, dass zwischen den verschiedenen innersekretorischen Organen Wechselbeziehungen bestehen. Was die Keimdrüsen anbetrifft, so sind Wechselbeziehungen zwischen ihnen und den Nebennieren, der Schilddrüse und Hypophysis gefunden worden. [Vgl. hierzu die kürzlich erschienene zusammenfassende Arbeit von Münzer (44)].

Unter meinen Beobachtungen habe ich dreimal auf die Schilddrüse (Fall 1,2 und 3) und zweimal auf die Hypophysis (Fall 2 und 3) mein Augenmerk gerichtet.

Die Schilddrüse war in Fall 1 auffallend klein. Sie war in Fall 2 nur undeutlich, in Fall 3 nicht mit Sicherheit zu fühlen, in beiden Fällen, wenn überhaupt vorhanden, zweifellos auch nur klein.

Dieser Befund steht in Analogie zu der Tatsache, dass eine Verkleinerung der Thyreoidea von Tandler und Gross (55) auch beim Kastraten festgestellt wurde und dass diese Autoren bei männlichen Eunuchoiden die Schilddrüse "niemals vergrössert", sondern "in der Regel schwer oder garnicht tastbar" fanden.

zunehmen, dass sie dann gerade den rudimentären heterosexuellen Anteil der Keimdrüsen verschont lassen, auf dessen Grundlage sich nun die neuen Sexualcharaktere entwickeln sollen. Aehnlich verbält es sich hinsichtlich des Klimakteriums. Kurt Mendel (42) hat kürzlich sehr interessante Mitteilungen über das, Klimakterium virile" gemacht, die dafür sprechen, dass ähnliche Ausfallserscheinungen wie bei Frauen etwa um die gleiche Zeit anch bei Männern eintreten, dass also, wie man danach annehmen muss, auch dje innere Sekretion der Hoden in einem gewissen Alter physiologischerweise nachlässt oder Veränderungen erfährt. Wenn sich auch die Möglichkeit nicht absolut bestreiten lässt, so ist es da-

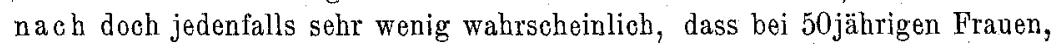
während die Ovarien funktionslos werden, ein in den Keimdrüsen angelegter rudimentärer Hodenanteil heterosexuello Charaktere (Bartwuchs oder männliche Kehlkopfbildung) nun noch zur. Entfaltung zu bringen vermag. 
Ein von Peritz (47) beschriebener weiblicher Eunuchoid hatte allerdings eine Struma. In diesem Falle bestand aber bemerkenswerterweise auch psychischer Infantilismus.

Anders verhält es sich mit der Hypophysis. Untersuchungen von Fichera (17), Cimoroni (14) sowie Tandler und Gross (55) haben - teils im Experiment, teils bei Skopzengezeigt, dass die Hypophysis nach der Kastration hypertrophiert. Dagegen konnte bei Eunuchoiden weder von Tandler und Gross (56) nọch von Peritz (47) eine Vergrösserung der Hypophysis konstatiert werden, und ebenso war auch in den beiden ron mir untersuchten Fällen eine Vergrösserung der Hypophysis nicht zu finden. [Diese Untersuchung wurde nach dem Vorgange von Tandler und Gross mittels Röntgenaufnahmen der Schädelbasis vorgenommen, um festzustellen, ob eine Verbreiterung der Sella turcica vorhanden sei.]

Es scheint somit hinsichtlich des Verhaltens der Hypophysis eine deutliche Differenz zwischen Eunuchen and Eunuchoiden zu bestehen.

Tandler und Gross (56) halten allerdings trotz ihres negativen Befundes für wahrscheinlich, dass \#auch bei der genitalen Hypoplasie, beim Eunuchoid, hypophysäre Veränderungen, zum mindesten funktioneller Natur", vorhanden sind. Bewiesen ist die Richtigkeit dieser Annahme aber noch nicht; auch würde die Differenz der makroskopischen Befunde damit natürlich nicht beseitigt sein. Peritz (47) andererseits stellt die ebenfalls noch unbewiesene Hypothese auf, die Ursache der Hypophysenhypertrophie bei den Kastraten liege darin, dass die Keimdrüsen hier dem Körper plötzlich entzogen wurden; bei den Eunuchoiden dagegen unterbliebe die Hypertrophie des Organs, weil der Einfluss der Keimdrüsen von vornherein ausgeschaltet war.

Ich kann in der Differenz der Hypophysisbefunde bei den Eunuchen und Eunuchoiden vorläufig lediglich eine weitere Stütze erblicken für meine Behauptung, dass die Eunuchen und die Eunuchoide nicht in vollem Maasse in Parallele zu einander gestellt werden dürfen.

Bei den Kastraten können Veränderungen, die an den innersekretorischen Organen, mit Regelmässigkeit nach der Kastration auftreten, selbstverständlich nur eine direkte oder indirekte Folge der Exstirpation der Keimdrüsen sein. Bei den Eunuchoiden dagegen, wo es sich um eine komplizierte Krankheitsform handelt, 
ist es noch gànz unmọglich, den inneren Zusammenhang der Drüsenanomalien mit einiger" Sicherheit zu enthüllen.

Treffend sagt Falta (16): „Im Experiment können wir den A usfall einer Blutdrüse erzengen. Schon dadurch rufen wir einen sehr komplizierten Zustand hervor, indem wir nicht nur die Funktion anderer. Blutdrïsen dadurch beeinflussen, sondern auf die Dauer wohl auch die formativen Prozesse verändern. Diese experimentell erzeugten Symptomenkomplexe können aber an Mannigfaltigkeit durchaus noch nicht wetteifern mit den Bildern, wie sie uns die Erkrankungen der Blutdrüsen vorführen."

Hinsichtlich des Skeletbaues unserer Eunuchoide schliesslich sind drei Erscheinungen hervorzuheben, nämlich:

1) die oben erörterte Disporportion im Sinne der verhältnismässig langen Extremitäten,

2) das Offenbleiben der Epiphysenlinien weit hinaus über die gewöhnliche Zeit,

3) die Kleinheit des Skelets im ganzen; (in einem Falle konnte man von Zwergwuchs sprechen.)

Was zunächst die Disproportion anbetrifft, so findet sie sich bei Eunuchen und Eunuchoiden und, wie es scheint, iiberhaupt überall da, wo die Epiphysen ungewöhnlich lange offen bleiben. Sie ist also zweifellos wohl als die Folge eines übermässig langdanernden und dabei ungleichen Knochenwachstums anzusehen. Diese Disproportion ist (darin gebe ich Tandler und Gross natürlich ohne weiteres recht) als solche keine infantile Verbildung; sie ist vielmehr eine Begleit- und Folgeerscheinung, die sich bei gewissen Formen des Infantilismus herausbildet.

Das Offenbleiben der Epiphysenlinien zeigt sich, wie wir wissen, bei einer Reihe von Krankheiten, die mit Veränderungen der Funktion innersekretorischer Organe einhergehen. ${ }^{1}$ ) So ist die Erscheinung wohl auch bei den Eunuchoiden als eine Folge der fehlerhaften Fúnktion dieser Drüsen anzusehen, ohne dass es aber bisher möglich wäre, zu entscheiden, inwieweit der Funktionsausfall gerade der Keimdrüsen dabei direkt von Einfluss ist.

1) Nach Falta (16) kann die Persistenz der Fipiphysenlinien „durch dio verschiedensten Momente bedingt sein, so durch Kastration oder Hypoplasie der Keimdrüsen, so bei Vergrösserung der Hypophyse, wenn sich diese im. Kindesalter einstellt. Sie findet sich regelmässig bei Persistenz der Thymus." 
Für die Kleinheit des Skelets bzw. den Zwergwuchs schliesslich könnte gleichfalls ein eigentümliches Zusammenwirken verschiedener spezifischer Drüsen das ursächliche Moment bilden. ${ }^{1}$ ) Für wahrseheinlicher aber halte ich, dass die Kleinheit des Skelets eine primäre Anomalie ist und dass diese Individuen von vornherein in allen ihren Teilen nur klein angelegt waren. Wie aber der Eunuch infolge des langen Offenbleibens seiner Epiphysenlinien grösser wird, als er im normalen Besitze seiner Keimdrüsen geworden wäre, so wird beim Eunuchoiden angeborener Zwergwuchs durch dass Offenbleiben der Epiphysenlinien - da das Wachstum auch hier länger andauert, als gewöhnlich — natürlich etwas vermindert.

Unsere Patientin 3 konnte mit Bestimmtheit angeben, dass sie noch in vorgerücktem Alter gewachsen sei, eine Erscheinung, die bei ähnlichen Personen schon Joachimsthal (37) konstatiert hatte. Joachimsthal untersuchte, nachdem schon früher Schauta und Paltauf (vgl. die Angaben von Joachimsthal) knorpelige Epiphysen bei $Z$ wergen in vorgerücktem Alter festgestellt hatten, mittels Röntgenstrahlen die Mitglieder einer sogenannten Liliputanertruppe und fand ,für die Fälle von reinem Zwergwuchs die Möglichkeit des Offenbleibens der Knorpelfugen selbst weit hinaus über die gewöhnliche Altersgrenze" and damit für die betreffenden Personen die Fähigkeit, noch in vorgerücktem Alter an Körperlänge zuzunehmen. Bei 2 weiblichen, 36 Jahre alten Mitgliedern der Truppe, die $114 \mathrm{bzw} .116 \mathrm{~cm}$ gross waren, fand Joachimsthal aber die Epiphysenlinien schon geschlossen; das Verhalten dieser beiden Individuen bot „den anderen Mitgliedern der Truppe gegenüber auch insofern einen Unterschied, als sie seit dem 20. Lebensjahre regelmässig menstruiert waren." Diese letztere Beobachtung ist sehr bemerkenswert; denn sie zeigt, dass ein Offenbleiben der Epiphysenlinien nicht für den Zwergwuchs an sich typisch ist. Es dürfte sich in Joachimsthals Fällen mit Offenbleiben der Epiphysenlinien eben auch um Eunuchoide gehandelt haben, bei denen Zwergwuchs und Offenbleiben der Epiphysenlinien eine Teilerscheinung der allgemeinen mit Anomalien innersekretorischer Organe einhergehenden Erkrankung gewesen sind. -

Fragt man sich schliesslich nach der kausalen Genese der infantilistischen Eunuchoide, so liegt hier, wie bei anderen

1) Falta (16) macht darauf aufmerksam, dass ein und dieselbe Dis. position (Hypoplasie der Keimdrüse, Vergrösserung der Hypophyse) einmal zum Zwerg- ein andermal zum Riesenwuchs führen kann, je nachdem die Schilddrüse funktioniert, und zwar findet sich Insuffizienz der Schilddrüse in dieser Kombination beim kretinen Zwerg. Falta sieht die Schilddrüse daher als einen entscheidenden Falitor für das Wachstum, die Keimdrüsen als entscheidend für den Schluss der Epiphysen an. Aebnlich sprechen sich auch Tandler und Gross (54) aus. 
Missbildungen die Möglichkeit vor, dass die letzte Ursache der Entstehung der Krankheitsform in äusseren oder in inneren Momenten zu suchen ist.

Was die äusseren Ursachen anbetrifft, soweit sie im extrauterinen Leben eingewirkt haben könnten, so habe ich mich bei einem ziemlicb grossen Material von Fällen von Infantilismus geringeren Grạdes am weiblichen Geschlechtsapparat, - wie sie ja häufig vorkommen, - vielfach bemüht, zu ermitteln, ob sich irgend ein derartiges ätiologisches Moment auffinden liess.

Aber weder die Art der Ernährung nach der Geburt, noch der Aufenthalt anf dem Lande oder in der Stadt während der Jugend, noch Erkrankungen chronischer oder akuter Art im Kindesalter sind, soweit meine Erfahrungen reichen, mit einiger Sicherheit als ätiologische Faktoren des Infantilismus zu beschuldigen.

Im Gegensatz hierzu sagt allerdings Hegar (30): „Die Schädlichkeiten, welche den Infantilismus hervorbringen, sind zahlreich. Abgesehen von der Erblichkeit und den ans dem Fruchtleben in die Kindheit verschleppten Anfängen, sind der Mangel der Mutterbrust und die schlechte Ernährung und Pflege hervorzuheben. Dazu kommen die vielen akuten und chronischen Infektionskrankheiten, unter welchen Lues und Tuberkulose eine grosse Rolle spielen; in den Pubertätsjahren die viel besprochenen Mängel der Erziehung und Lebensweise" (siehe auch die Arbeiten von E. Kehrer (38) und von F. A. Kehrer (39) ${ }^{1}$ ).

Wenn Beziehungen zwischen Erkrankungen wie Tuberkulose und Infantilismus wohl zweifellos bestehen, so dürfte die Tuberkulose nicht als die Ursache des Infantilismus anzusehen sein; viel eher dürfte der Infantilismus eine Disposition für die Entstehung der Tuberkulose abgegeben haben, wie dies ja auch der Lehre von W. A. Freund (21) entspricht.

Mit dieser Auffassung ganz in Uebereinstimmung stehen die bemerkenswerten Ergebnisse, zu denen kürzlich auf Grund pathologisch-anatomischer Untersuchungen über die Entwickelungsstörungen der männlichen Keimdrüsen im Kindesalter Kyrle (40) gelangt ist. Kyrle wies u. a. nach, dass eine nicht unbeträchtliche Zahl von Knaben mit unentwickelten Keimdrüsen geboren wird.

Noch viel weniger als bei den geringeren Graden des

1) Die von E. Kehrer (38) hervorgehobene Tatsache, dass Campbel durch einseitige Fleisohfütterung bei Ratten Entwickelungsstörungen des Uterus experimentell hervorgerufen hat, ist allerdings sehr beachtenswert. $\mathrm{Ob}$ Aehnliches beim Menschen vorkommt, bedarf aber noch des Beweises. 
Infantilismus dürfte bei der hochgradigen Form der Eunuchoide die kausale Genese in das extrauterine Leben zu verlegen sein.

Ob aber im intrauterinen Leben äussere Momente ätiologisch eingewirkt haben, oder ob es sich um primäre Keimesvariationen handelt, entzieht sich noch der Entscheidung.

Denkbar wäre, dass die Entstehung der infantilistischen: Eunuchoide auf intrauterine Stoffwechselanomalien zurückzuführen. ist. So sagt E. Sehwalbe (50): „Die embryonale und postfötale Entwickelungshemmung könnte vielleicht mit dem Stoffwechsel im weitesten Sinne in Zusammenhang gebracht werden." E. Kehrer (38) ferner hat mit Recht auf die Analogie mit den Arbeiterinnen der Bienen hingewiesen. Derartige physioIogische Eunuchoide - so darf ich die Arbeiterinnen der Bienen wohl nennen - gehen bekanntlich aus Eiern der gleichen Art wie die Bienenkönigin hervor, werden aber von den Bienen im Larvenzustande mit anderem Futter aufgezogen. ${ }^{1}$ )

Andererseits spricht aber, meiner Ansicht nach, die häufige Kombination des Infantilismus mit sonstigen Anomalien - speziell mit hermaphroditischen Merkmalen - für eine sehr frühe kausale Genese der ganzen Missbildung und damit wohl am meisten für die Annahme primärer Keimesvariationen. --

Was nun endlich das vorzeitig entwickelte Kind anbetrifft, so will ich mich darüber, unter Hinweis auf eine kürzlich erschienene ausführliche Arbeit Neuraths (46) über ,die vorzeitige Geschlechtsentwickelung:", kurz fassen.

Das Interesse, das der Fall hier darbietet, liegt hauptsächlich. in dem genauen Gegensatz, in dem die Symptome bei dem Kinde zu denen bei den Eunuchoiden stehen.

Wir finden bei dem Kinde eine vorzeitige Entwickelung der Genitalien mit vorzeitiger Menstruation und dabei vorzeitige Entwickelung sekundärer Geschlechtscharaktere, Wachstum des ganzen Körpers über das dem Alter entsprechende Maass hinaus, sodass das 4 jährige Kind die Grösse eines 8-9 jährigen erreicht hatte, und,

1) "Ohne allen Zweifel, " sagt Biedermann (6), ,ist die geschilderte Verschiedenbeit in der Zusammensetzung des Larvenfutters (Mitteldarminhaltes der Arbeitsbienen) auch ein ausschlaggebender Falitor bei der Geschlechtsbestimmung; denn es ist bekannt, dass nach Verlust einer Königin die Bienen aus Arbeitslarven eine nene Königin erziehen, indem sie die Zelle, in der die Entwickelung vor sich geht, vergrössern und ,Königinfutterbrei ${ }^{6}$ verabreichen." 
was besonders interessant ist, eine weit vorgeschrittene Entwickelung des Knochensystems.

Wie ich der Arbeit Neuraths (46) entnehme, liegt eine Röntgenuntersuchung über die Knochenverhältnisse bei vorzeitiger Geschlechtsreife bisher nur in 3 Fällen vor. Die Untersuchung ergab bei einem vorzeitig entwickelten Knaben von $5^{\frac{1}{1} / 2}$ Jahren Verhältnisse wie sonst bei 15jährigen (Fall Linser), bei einem $51 / 2$ jährigen Knaben (Fall von Hudovernig und Popovils) Verhältnisse wie sonst bei 15-16jährigen und in einer Beobachtung von Neurath selbst bei einem 6jührigen frïhreifen Mädchen Verhältnisse, die dem 11. Jahre entsprachen. ${ }^{1}$ )

Auch bei dieser Abnormität handelt es sich vermutlich nicht um isolierte Veränderungen an den Keimdrüsen, vielmehr sind. wahrscheinlich auch hier gewöhnlich mit einer fehlerhaften Funktion und Beschaffenheit der Eierstöcke von vornherein noch andere Bildungsanomalien verbunden.

So findet man, ganz ähnlich wie dies beim Infantilismus nicht selten der Fall ist (siehe oben Seite 567, 568), auch in den Beobachtungen von vorzeitiger Geschlechtsreife zuweilen sehr ausgesprochene Symptome von heterosexuellem Typus ausgeprägt (siehe die Zusammenstellung von Fällen bei Neurath).

Lesser (40a) zum Beispiel beobachtete ein 6 jähriges vorzeitig menstruiertes Mädchen. Das Kind hatte äusserst kräftigen Körperbau, stark entwickelte Mammae, schwarzen Backenbart; die Oberlippe war mit kurzen dünnen dunklen Haaren dicht besetzt, der übrige Körper war, mit Ausnahme der Hände und Füsse, stark behaart, besonders auch in der Schamgegend, an der Linea alba und in der Achselhöhle.

Neurath erwähnt auch, dass in der grossen Zahl der Beobachtungen die übermässige Körperentwickelung bereits bei der Geburt aufgefallen ist und dass schon Kussmaul solche Fälle für die Auffassung der Frühreife als einer bereits intrauterin beginnenden Abnormitat verwertet hat. In unserer Beobachtung soll die Hebamme bei der Geburt des Kindes bemerkt haben, dasselbe sei "wie ein Vierteljahrskind".

Dass schon im intrauterinen Leben eine vorzeitige Körperentwickelung zugleich mit Anomalien innersekretorischer Organe vorkommt, habe ich (62) in einem Falle direkt nachweisen können: Es handelte sich um ein intra partum abge-

1) Anmerkung bei der Korrektur: Im Anschluss an meinen Vortrag über das vorliegende Thema (s. Seite 542 Anm.) hat Nacke (44a) bei einem früher von ihm mitgeteilten Falle von vorzeitiger Geschlechtsentwicklung bei einem.Mädchen eine Röntgenuntersuehung vornehmen lassen und dabei ebenfalls eine weit vorgeschrittene Entwicklung des Skeletbaues konstatiert. 
storbenes, nicht übertragenes Riesenkind mit einem Gewicht von 5480 g. Das Herz dieses Neugeborenen hatte eine Grösse, wie sie sonst erst mit 11/2 Jahren erreicht wird; dagegen waren Thymus und Thyreoidea minimal entwickelt. -

Am Schlusse meiner Ausführungen möchte ich noch kurz folgendes bemerken:

Selbstverständlich sind mit den hier erörterten Anomalien die pathologischen Befunde in diesen Fällen nicht erschöpft. So würde wohl der Beckentypus, die Form des Thorax, die Beschaffenheit des Kehlkopfes und vieles andere noch eines besonderen Studiums wert gewesen sein.

Ich betone ferner, dass ich mir der Schwierigkeiten wohl bewasst bin, die bestehen, wenn man aus Fällen wie die meinen, ohne die Möglichkeit pathologisch-anatomischer Untersuchung, Schlüsse zieht. Wenn ich trotzdem diese Beobachtungen im Zusammenhang mitteilen zu dürfen glaubte, so geschah es, weil die Fälle selten sind und weil meine Befunde, wie ich denke, zu weiteren Studien anregen müssen, ist doch, um mit einem Worte unseres Altmeisters Hegar (30) zu schliessen, die "Lehre vom Fötalismus und Infantilismus ein weiterer Anknüpfungspunkt unserer Geburtshilfe und Gynäkologie an die Entwickelungsgeschichte, Biologie und Vererbungslehre."

\section{Li t e r a t ur.}

1. Alterthum, Die Folgezustände nach Kastration und die sekundären Geschlechtscharaktere. Beiträge z. Geburtsh. u. Gyn. 1899. Bd. 2.

2. Bartel, Ueber die bypoplastische Konstitution und ihre Bedentung. Wiener klin. Wochenschr. 1908.

3. Bartel und Herrmann, Ueber die weibliohe Keimdrüse bei Anomalie der Konstitution. Monatsschr. f. Geburtsh. u. Gyn. 1911. Bd. 33.

4. Bayer, Vorlesungen über allgemeine Geburtshilfe. Strassburg 1908. Bd. 1.

5. Bendix, Lehrbuch der Kinderheilkunde. 1903. 3. Aufl. S. 7.

6. Biedermann, Die Aufnahme, Verarbeitung und Assimilation der Nahrung. In Wintersteins Handbuch der vergleichenden Physiologie. 1910. Bd. 2.

7. Biedl, Innere Sekretion. Ihre physiologisohen Grundlagen und ihre Bedeutung für die Pathologie. Urban und Schwarzenberg. 1910.

8. Blumreich, Diskussion zum Vortrag von Bunge. Zeitschr. f. Geburtsh. u. Gyn. 1906. Bd. 57. 
9. Bresca, Experimentelle Untersuchungen über die sekundären Sexual: charaktere der Tritonen. Archiv für Entwickelungsmechanik. 1910. Bd. 29.

10. Bunge, Defekt der inneren Genitalien. Verhandl, der Gesellsch. für Geburtsh. u. Gyn. yom 26. Januar 1906. Zeitschr. f. Geburtsh. u. Gyn. 1906. Bd. 57.

11. Burrage, Amer. Journ. of med. science 1897. Vol. CXIII, Zitiert nach Büttner.

12. Büttner, Zur Lebre von der rudimentären Entwickelung der Müllerschen Gänge. Beitr. z. Geburtsh. u. Gyn. 1909. Bd. 14.

13. Chrobak and v. Rosthorn, Die Erkrankungen der weiblichen Geschlechtsorgane. II. Teil: Die Missbildungen der weiblichen Geschlechtsorgane. Wien u. Leipzig 1908. Hölder.

14. Cimoroni, Lo sperimentale 61. 1907. Arch. ital. de biol. 48. 1908. Zitiert nach Tandler und Gross.

15. Durkworth, Notes on the anatomy of an eunuchoid man. Journ. of. Anat. and. Physiol. 41. Zitiert nach Tander und Gross.

16. Falta, Ueber die Korrelation der Drüsen mit innerer Sekretion. Ergebnisse der wissenschaftlichen Medizin. Leipzig 1909. H. 3.

17. Fichera, Sulla ipertrofia della ghiandola pituitaria consecutiva alla castrazione. Policlinico 1905. Zitiert nach Tandler und Gross.

18. Franz, Zur Entwickelung des knöchernen Beckens nach der Geburt. Beitr. z. Geburtsh. u. Gyn. 1909. Bd. 13.

19. Freund, W. A, Ueber dio Indikation zur operativen Behandlung der erkrankten Tuben. Volkmanns Samml. klin. Vortr. 1888. No. 323.

20. Freund, W. A., Zur Anatomie, Physiologie und Pathologie der Douglastasche. Beitr. z. Geburtsh. u. Gyn. 1899. Bd. 2.

21. Freund, W. A., Thoraxanomalien als Prädisposition zu Lungenphthise und Emphysem. Berliner klin. Wochenschr. 1902.

22. Fromme, Ueber die infantilen Störungen beim weiblichen Geschlecht. Med. Klinik. 1910. No. 41.

23. Griffith, The condition of the testes and prostata gland in eunuchoid persons. Journ. of anatomy. 1894. Vol. 28. Zitiert nach Tandler und Gross.

24. Gruber, Wenzel, Die kongenitale Anorchie beim Menschen. Medizin. Jahrbücher. 1868. Bd. XV. Zitiert nach Sellheim.

25. Halban, Die Entstehung der Geschlechtscharaktere. Dieses Archiv. 1903. Bd. 70.

26. v. Hansemann, Descendenz und Pathologie. Berlin 1909. Hirschwald.

27. Hegar, A., Kastration bei Frauen. Volkmanns Samml. klin. Vorträge. 1876.

28. Hegar, A., Abnorme Behaarung und Uterus duplex. Beitr. z. Geburtsh. u. Gyn. 1898. Bd. 1.

29. Hegar, A., Korrelationen der Keimdrüsen und Geschlechtsbestimmung. Beitr. z. Geburtsh. u. Gyn. 1903. Bd. 7.

30. Hegar, A., Entwickelungsstörungen, Fötalismas und Infantilismus. Münchener med. Wochenschr. 1905. No. 11. 
31. Hegar, A., Entwickelungsstörungen. Deutsche med. Wochenschr. 1910. No. 40.

32. Helmbold, In Neugebauers Werk (45). S. 235.

33. Herbst; Formative Reize in der tierischen Ontogenese. Leipzig 1901.

34. Herrmann, Demonstration von Ovarien beim Status lymphaticus, bzw. hypoplasticus. Centralbl. f. Phys. 1909. Bd. 23. No. 8.

34a. Heyn, Pseudohermaphroditismus masculinus completus. Zeitschrift für Geburtsh. u. Gyn. 1910. Bd. 65.

35. Hocheisen, Diskussion zum Vortrag vion Bunge. Zeitschr. f. Geburtsh. u. Gyn. 1906. Bd. 57.

36. Holzbach, Die Hemmungsbildungen der Mällerșchen Gänge im Lichte der vergleichenden Anatomie und Entwickelungsgeschichte. Beitr. z. Geburtsh. u. Gyn. 1909. Bd. 14.

37. Joachimsthal, Ueber Zwergwuchș und yerwandte Wachstumsstörungen. Deutsche med. Wochenschr. 1899.

38. Kehrer, E., Die Entwickungsstörungen beim weiblichen Geschlecht. Beitr. z. Geburtsh. u. Gyn. 1910. Bd. 15.

39. Kehrer, F. A., Die Ursachen des Infantilismus. Beitr. z. Geburtsh. u. Gyn. 1910. Bd. 15.

40. Kyrle, Ueber Entwickelungsstörungen der männlichen Keimdrüsen im Jugendalter. Wiener klin. Wochenschr. 1910. Nr. 49.

40a. Lesser, Ein Fall von Hypertrichosis universalis und frühzeitiger Geschlechtsreife. Zeitschr. f. klin. Med. Bd. 41. 1900.

41. Mayer, A., Ein Beitrag zur Lehre von der Hypoplasie der Genitalien und vom Infantilismus auf Grund von klinischen Beobachtungen. Beitr. z. Geb. u. Gyn. Bd. 12. 1908.

42. Mendel, Kurt, Die Wechseljabre des Mannes. Neurolog. Centralbl. 1910.

43. Menge, Bildungsfehler der weiblichen Genitalien. In Veits Handbuch d. Gyn. 2. Aufl. Bd. 6. 2, Hälfte. 1910.

44. Münzer, Ueber die innere Sekretion der Keimdrüsen. Berlin. klin. Wochenschr. 1910.

44a. Nacke, Sitzungsbericht d. Gesellsch. f. Geburtsh. u. Gyn. zu Berlin vom 24. März 1911.

45. Neugebauer, Hermaphroditismus beim Menschen. Leipzig. 1908.

46. Neurath, Die vorzeitige Geschlechtsentwickelung. Ergebnisse d. inn. Med. u. Kinderheilk. Bd. 4. 1909.

47. Peritz, Ueber Eunuchoide. Neurolog. Centralbl. 1910.

48. Pick, L., Ueber Neubildungen am Genitale bei Zwittern usw. Dieses. Arehiv. Bd. 76. 1905.

49. Schwalbe, Ernst, Morphologie d. Missbildungen des Menschen und der Tiere. I. Teil. Allgem, Missbildungslebre. Jena 1906.

50. Schwàlbe, Ernst, Dasselbe. III. Teil. Die Einzelmissbildungen. 1. Abt. S. 25. Jena 1909.

51. Sellheim, Kastration und Knochenwachstum. Beitr. z. Geb. u. Gyn. Bd. 2. 1899.

52. Still er, Magengeschwür und Tungentuberkulosẹ. Berl, klin. Wochenșchr. 1911. Nr. 8. 
53. Strassmann, P., Demonstration eines Hermaphroditen. Verhandl. d. Ges. f. Geb. u. Gyn. zu Berlin vom 25. Oktober 1909. Stuttgart 1910.

54. Tandler und Gross, Einfluss der Kastration auf den Organismus. Wiener klin. Wochenschr. 1907.

54a. Tandler und Gross, Untersuchungen an Skopzen. Wiener klin. Wochenschr. 1908. Nr. 9.

55. Tandler und Gross, Ueber den Einfluss der Kastration auf den Organismus. II. Die Skopzen. Arch. f. Entwickelungsmechanik. Bd. 30.1910.

56. Tandler und Gross, Ueber den Einfluss der Kastration auf den Organismus. III. Die Eunuchoide. Arch. f. Entwickelungsmechanik. Bd. 29. 1910.

57. Tandler und Keller, Ueber den Einfluss der Kastration auf den Organismus. IV. Die Körperform der weiblichen Frübkastraten des Rindes. Arch.

f. Entwickelungsmechanik. Bd. 31. 1910.

58. Virchow, R., Vorstellung eines Hermaphroditen, Berliner klin. Wochenschr. 1872. Nr. 49.

59. Virohow, R., Diskussionsbemerkung. Berliner klin. Wochenschr. 1898. S. 178 .

60. Wilms and Sick, Die Entwickelung der Knochen der Extremitäten von der Geburt bis zum vollendeten Wachstum. 'In Archiv und Atlas der normalen und pathologischen Anatomie in typischen Röntgenbildern. Hamburg 1902.

61. v. Winckel, Ueber die Einteilung, Entstehang rund Benennung derBildungsbemmungen der weiblichen Sexualorgane. Samml. klin. Vortr. Nr. 251 u. 252. 1899:

62. Wolff, Bruno, Demonstration eines neugeborenen Kindes von abnorm starker Entwickelung. Zeitschr. f. Geb. u. Gyn. Bd. 45. H. 2. 1901.

\section{Erklärung der Abbildnngen.}

Fig. 1: Patientin Fall 1.

Fig. 2: Röntgenbild der rechten Hand der Patientin Fall 1. Offene. Epiphysenlinien an den Phalangen und Metacarpalknochen, an der unteren Ulna- und Radiusepiphyse. Das Bild entspricht. Verhältnissen wie sonst im 15. Lebensjahr. (Die Röntgenbilder der Hände der Patientinnen 2 und 4 sind ganz ähnlich.)

Fig. 3: Röntgenbild der rechten Hand der Patientin Fall 3. Offene Epiphysenlinien an der unteren Radius- und Ulnaepiphyse. Das Bild ent'spricht Verhältnissen wie sonst im 18. Lebensjahre.

Fig. 4: Röntgenbild der rechten Hand des 4jährigen Kindes (Fall 5): Das Bild entspricht Verhältnissen wie soust im 10. Lebensjahre. 